\title{
Two-Stage Change Detection for Synthetic Aperture Radar
}

\author{
Miriam Cha, Member, IEEE, Rhonda D. Phillips, Member, IEEE, \\ Patrick J. Wolfe, Member, IEEE, and Christ D. Richmond, Senior Member, IEEE
}

\begin{abstract}
Coherent change detection using paired synthetic aperture radar images is often performed using a classical coherence estimator that is invariant to the true variances of the populations underlying each paired sample. While attractive, this estimator is biased and requires a significant number of samples to yield good performance. Increasing sample size often results in decreased image resolution. Thus, we propose use of Berger's coherence estimate because with the same number of pixels, the estimator effectively doubles the sample support without sacrificing resolution when the underlying population variances are equal or near equal. A potential drawback of this approach is that it is not invariant since its distribution depends on the pixel pair population variances. While Berger's estimator is inherently sensitive to the inequality of population variances, we propose a method of insulating the detector from this acuity. A two-stage change statistic is introduced to combine a non-coherent intensity change statistic given by the sample variance ratio followed by the alternative Berger estimator which assumes equal population variances. The first stage detector identifies pixel pairs that have non-equal variances as changes caused by the displacement of sizable object. The pixel pairs that are identified to have equal or near equal variances in the first stage are used as an input to the second stage. The second stage test uses the alternative Berger coherence estimator to detect subtle changes such as tire tracks and footprints. We show experimentally that the proposed method yields higher contrast SAR change detection images
\end{abstract}

This work is sponsored by the United States Air Force under Air Force Contract FA8721-05-C-0002. Opinions, interpretations, conclusions and recommendations are those of the author and are not necessarily endorsed by the United States Government. Approved for public release; distribution is unlimited.

M. Cha and C.D. Richmond are with MIT Lincoln Laboratory, 24 Wood Street, Lexington, MA 02420. (e-mail: \{miriam.cha,christ\}@11.mit.edu)

R.D. Phillips is with Microsoft, 1 Memorial Drive, Cambridge, MA 02142. (e-mail: rhonda.phillips@microsoft.com)

P.J. Wolfe is with the Department of Statistical Science, University College London, London WC1E 6BT, UK. (e-mail: p.wolfe@ucl.ac.uk) 
than the classical coherent change detector (state of the art), alternative coherent change detector, and intensity change detector. Experimental results are presented to show the effectiveness and robustness of the proposed algorithm for SAR change detection.

\section{INTRODUCTION}

Synthetic aperture radar (SAR) is an important modality in remote sensing due to its ability to form high resolution images with relative invariance to weather and lighting conditions. SAR images are formed using a moving radar that collects data over a scene from multiple perspectives. The resulting data are complex-valued, with the magnitude corresponding to the reflected signal intensity of the scene and the phase indicating scattering properties.

One application of SAR is change detection, which utilizes two SAR data collections of the same scene at different times to infer changes that have occurred in between data collections [1]. SAR change detection algorithms can be categorized into two: 1) non-coherent intensity change detection utilizing local changes in SAR magnitude images to indicate large-scale changes, such as the appearance of a sizeable object during the second collection that was not present during the first; and 2) coherent change detection (CCD) that uses SAR phase as well as magnitude to estimate the coherence between the two SAR images. CCD requires the two image collections to use identical collection geometries, so that each respective image phase is aligned, leading to the detection of smaller-scale changes, such as those made by a vehicle driving on a soft surface [2] [3]. Additionally, some work [4] [5] consider change detection between polarimetric acquisitions since polarimetric analysis can enhance discrimination capability.

As [6]-[8] have investigated, the traditional coherence magnitude estimator is biased, particularly when the true coherence is small. This bias can be reduced by an increase in the number of samples. However, in practice, there are a limited number of samples to be obtained from each spatial location in a pair of SAR images, as they must be "borrowed" from a local neighborhood or spatial window. As the number of neighboring pixels used to estimate coherence is increased, the effective spatial resolution of the resulting CCD image is decreased, making detection of small-scale changes more difficult. Furthermore, as the size of the sample window increases, the assumption that the samples are drawn independently from the same distribution is less likely to be met. Accurate estimation of coherence from a limited number of samples is a challenging problem, which must be overcome either through better models for the data or more accurate 
estimators.

Here we adopt the latter approach and introduce a two-stage method that leverages both noncoherent and coherent algorithms for SAR change detection. Similar to the approach in [9], we jointly use intensity and coherence characteristics of SAR images. First, non-coherent intensity change detection is employed to identify large-scale changes. Then coherent change detection using a coherence estimator is utilized to detect small-scale changes. Traditionally, the classical estimator based on the Pearson correlation coefficient is used for coherence, however, [10] shows that with reasonable assumptions, an alternative coherence estimator yields better coherence estimation and change detection performance. This alternative coherence estimator assumes both populations have equal variances, which is more likely after the application of the first test. In this work, we perform further analysis to characterize the performance of the alternative coherence estimator, and analyze its performance within the two-stage change detection procedure. An exact joint probability density function for the statistics of the two-stage CCD is derived. The focus of this initial study, however, will be the development of a heuristic approach to threshold selection and the presentation of encouraging results from empirical data analysis due to the integral of the joint density function being non-trivial to be solved analytically.

The organization for the remainder of the paper is as follows. In Section II, we provide a detailed formulation of the intensity change detection, the traditional coherent change detection and the alternative coherent change detection. Section III derives the new two-stage change detection technique that utilizes intensity change detection in the first stage and the alternative coherence statistic in the second stage. As we will focus on a heuristic approach to the two-stage change detection, in Section IV, we discuss statistical effects of combining the first and the second stage tests. Section V contains numerical and experimental results to show the effectiveness of the proposed algorithm for SAR change detection, and Section VI concludes the paper.

\section{Change Detection}

SAR data is often assumed to be collections of spatially uncorrelated pixels drawn from a zero-mean circularly complex Gaussian distribution. The statistic used to estimate change in SAR data sets, corresponding to the estimated coherence between a pair of SAR observations, is thus a random variable depending on the true underlying coherence as well as the number of samples employed in coherence estimation. 
Given two spatially registered SAR data sets, $f$ and $g$ of $N$ pixels, one can form a joint data vector $X=[f, g]^{T} \in \mathbb{C}^{N \times 2}$. The $N$ sample pairs of observations, $X_{k}=\left[f_{k}, g_{k}\right], k=1, \ldots, N$, are viewed as independent samples from a zero-mean, bivariate, complex Gaussian distribution with covariance matrix

$$
\Sigma=\mathbb{E}\left(X X^{H}\right)=\left[\begin{array}{cc}
\sigma_{f}^{2} & \rho \sigma_{f} \sigma_{g} \\
\bar{\rho} \sigma_{f} \sigma_{g} & \sigma_{g}^{2}
\end{array}\right]
$$

where

$$
\sigma_{f}^{2}=\mathbb{E}\left(|f|^{2}\right), \quad \sigma_{g}^{2}=\mathbb{E}\left(|g|^{2}\right), \quad \rho=\frac{\mathbb{E}\left(f g^{H}\right)}{\sqrt{\mathbb{E}\left(|f|^{2}\right) \mathbb{E}\left(|g|^{2}\right)}}
$$

Here $\rho$ is the complex correlation coefficient, and $\bar{\rho}$ denotes its complex conjugate. The covariance matrix $\Sigma$ is typically estimated by the maximum likelihood estimator,

$$
\frac{1}{N} \sum_{k=1}^{N} X_{k} X_{k}^{H}=\frac{1}{N}\left[\begin{array}{cc}
\sum_{k}\left|f_{k}\right|^{2} & \sum_{k} f_{k} g_{k}^{H} \\
\sum_{k} f_{k}^{H} g_{k} & \sum_{k}\left|g_{k}\right|^{2}
\end{array}\right] \equiv\left[\begin{array}{cc}
\hat{\sigma}_{f}^{2} & \hat{\rho} \hat{\sigma}_{f} \hat{\sigma}_{g} \\
\hat{\bar{\rho}} \hat{\sigma}_{f} \hat{\sigma}_{g} & \hat{\sigma}_{g}^{2}
\end{array}\right] \triangleq \frac{1}{N} A,
$$

whose distribution is given by a bivariate complex Wishart density [11],

$$
p(A ; \Sigma, N)=\frac{|A|^{N-2} \exp \left(-\operatorname{tr}\left(\Sigma^{-1} A\right)\right)}{\pi \Gamma(N) \Gamma(N-1)|\Sigma|^{N}} .
$$

The function $p(A ; \Sigma, N)$ is defined over the domain where $A$ is Hermitian positive definite. In practice, $A$ is obtained using a spatial window of $f_{k}$ and $g_{k}$ in the respective SAR images. The following subsections describe the classical methods of SAR change detection assuming this statistical model of the SAR data.

\section{A. Non-coherent Intensity Change Detection}

Intensity based change detection between remote sensing images is often achieved using one of two operations: subtracting the two images to identify change as a large difference, or dividing the two images to identify change as a quotient that significantly deviates from unity [12] [13]. SAR change detection uses the quotient approach [14]: change is estimated using the result of dividing one magnitude image by the other. Specifically, a variance at one location in $f$ is estimated using a spatial window and the corresponding variance of $g$ is estimated, and the change statistic is

$$
\hat{R}=\frac{\hat{\sigma}_{f}^{2}}{\hat{\sigma}_{g}^{2}}
$$


Values of $\hat{R}$ that differ substantially from one are labeled as change. This statistic is frequently used to test if the underlying variances of two populations are different. The probability density function of $\hat{R}$ is given by [15]:

$$
p(\hat{R} ; R,|\rho|, N)=\frac{\hat{R}^{N-1}(\hat{R}+R) R^{N}\left(1-|\rho|^{2}\right)^{N}}{B(N, N)\left[(\hat{R}+R)^{2}-4 R \hat{R}|\rho|^{2}\right]^{N+1 / 2}}, \quad \hat{R} \geq 0
$$

where $B(N, N)$ is the beta function and $R=\sigma_{f}^{2} / \sigma_{g}^{2}$, i.e. the ratio of the true variances.

The problem of detecting different population variances is formulated as a hypothesis test, where the null hypothesis is that the two population variances are equal and the alternative hypothesis is that those populations have different variances. In the absence of correlation, the sample variance ratio $\hat{R}$ is proportional to a central $F_{2 N, 2 N}$ distribution if the null hypothesis $\sigma_{f}=\sigma_{g}$ is true, making this test an $F$-test. Therefore, if the null hypothesis is true and $\rho=0$,

$$
F\left(R \mid H_{0}\right) \equiv P\left(\hat{R} \leq R \mid H_{0}\right)=I_{R /(1+R)}(N, N)
$$

where $I_{v}(a, b)$ is the incomplete beta function [16]. For a chosen test significance level $\alpha$, the upper critical value $R_{u, \alpha}$ and the lower critical value $R_{l, \alpha}$ of the $F_{2 N, 2 N}$ distribution can be found such that

$$
F\left(R_{u, \alpha} \mid H_{0}\right)=1-\frac{\alpha}{2}, \quad F\left(R_{l, \alpha} \mid H_{0}\right)=\frac{\alpha}{2} .
$$

A sample value $\hat{R}$ such that $\hat{R}<R_{l, \alpha}$ or $\hat{R}>R_{u, \alpha}$ results in the null hypothesis being rejected at significance level $\alpha$. When applied to SAR change detection, rejection of the null hypothesis indicates change. This test will reveal large-scale changes that affect SAR magnitude values, such as a car that appears in one image but not another. Smaller scale change detection requires a different change detection method. It will be shown that changes such that $\sigma_{f} \neq \sigma_{g}$ can occur for a wide range of coherence values $|\rho| \in[0,1]$. Those that correspond to large scale changes are more likely when $|\rho|=0$. This will be the basis for the method of threshold selection for $\hat{R}$ and is discussed in more detail in Section IV-B.

\section{B. Classical Coherent Change Detection}

While non-coherent change detection is generally applicable to any type of real-valued image, coherent change detection is applicable to complex data and exploits both magnitude and phase. The additional phase data allows smaller scale changes to be detected, such as tire imprints on soft soil. The small ground surface change affects radar scattering, which affects phase. The 
parameter that is often used to indicate this type of change is coherence $\rho$, which is often estimated using a classical coherence estimator,

$$
\hat{\rho}_{c}=\frac{A_{12}}{\sqrt{A_{11}} \sqrt{A_{22}}} .
$$

Denote that $A=\left[A_{11}, A_{12} ; A_{21}, A_{22}\right]$ in (3). The statistic $\left|\hat{\rho}_{c}\right|$ is a random variable distributed according to

$$
p\left(\left|\hat{\rho}_{c}\right| ;|\rho|, N\right)=2(N-1)\left(1-|\rho|^{2}\right)^{N}\left|\hat{\rho}_{c}\right|\left(1-\left|\hat{\rho}_{c}\right|^{2}\right)^{N-2}{ }_{2} F_{1}\left(N, N ; 1 ;|\rho|^{2}\left|\hat{\rho}_{c}\right|^{2}\right),
$$

where ${ }_{2} F_{1}(\cdot, \cdot ; \cdot ; \cdot)$ is the Gauss hypergeometric function [6]. This distribution is invariant to $\sigma_{f}$ and $\sigma_{g}$, and only depends on the true underlying coherence magnitude $|\rho|$ and number of samples used in estimation $N$. Examples of this distribution for different values of $|\rho|$ and $N$ are shown as a blue line in Fig. 1. Notice that the bias of the estimator increases with decreasing $|\rho|$, and is especially pronounced when $N$ is small. Since the number of samples available for coherence estimation is often small in practice, this bias could affect change detection by decreasing true positives.

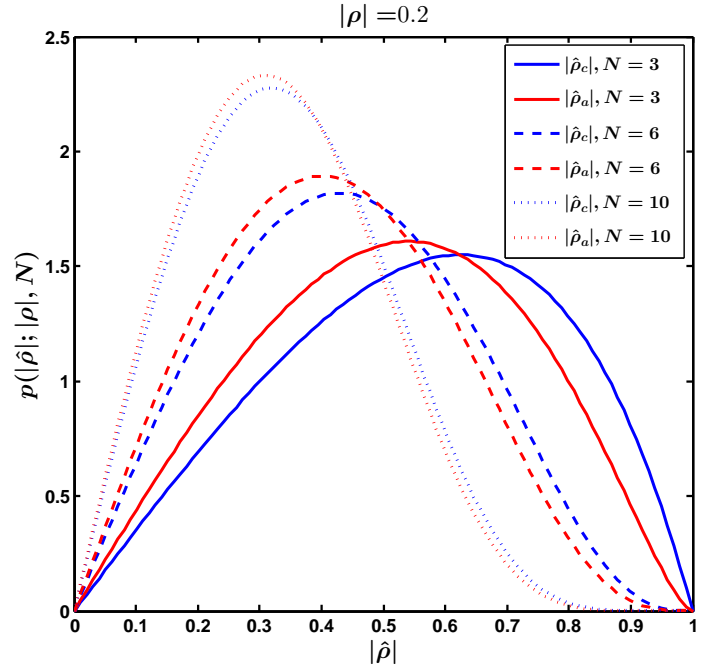

(a)

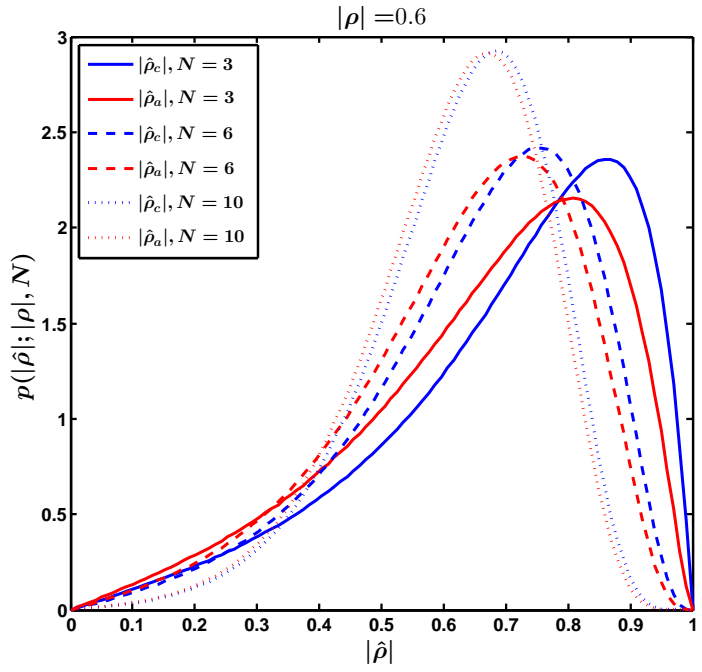

(b)

Fig. 1: Sampling distributions of $\left|\hat{\rho}_{c}\right|$ (blue) and $\left|\hat{\rho}_{a}\right|$ (red) shown as a function of sample size $N$, for true parameter values $\sigma_{f}=\sigma_{g}$, (a) $|\rho|=0.2$ and (b) $|\rho|=0.6$. 
Note that as a single pair of SAR images is assumed available, only a limited number of samples is available to estimate coherence. The size of the spatial neighborhood can be increased to increase the effective number of samples. This can have unintended effects though, such as smoothing the eventual CCD image and resulting in missed change detection, especially for subtle ground changes. However, using a coherence estimator capable of exploiting a near equal variance scenario has the potential to effectively double the number of samples available for estimation. Figure 1 shows that this could have benefits especially in the very low sample support cases, e.g. $N \leq 5$.

\section{Berger's Alternative Coherent Change Detection}

An alternative coherence estimator, introduced by Berger [7], that assumes variance equality is explored as a means of improving change detection performance. As change detection requires two SAR images of the same scene, the underlying variances will be near equal absent significant changes. When this equal variance assumption is met, the natural estimator of the complex correlation coefficient $\rho$, denoted $\hat{\rho}_{a}$, can be written as a function of the elements of $A$ as

$$
\hat{\rho}_{a}=\frac{2 A_{12}}{\left(A_{11}+A_{22}\right)} .
$$

Notice that the denominator contains a sum rather than a product of two random variables, suggesting a more stable estimator. Furthermore, since this estimator assumes both variance terms are equal, the number of samples used to estimate the true variance is potentially doubled. In scenes where most of the underlying variance remains unchanged, this estimator can be expected to offer improved properties over the classical estimator $\hat{\rho}_{c}$ of (9).

The expression for the probability density function of estimated coherence magnitude $\left|\hat{\rho}_{a}\right|$ is derived by several authors, including in [7]:

$$
p\left(\left|\hat{\rho}_{a}\right| ;|\rho|, N\right)=(2 N-1)\left(1-|\rho|^{2}\right)^{N}\left|\hat{\rho}_{a}\right|\left(1-\left|\hat{\rho}_{a}\right|^{2}\right)^{N-\frac{3}{2}}{ }_{2} F_{1}\left(N, N+1 / 2 ; 1 ;|\rho|^{2}\left|\hat{\rho}_{a}\right|^{2}\right) .
$$

This assumes $\sigma_{f}=\sigma_{g}$ and only parameterized by $|\rho|$ and $N$. Examples of the distribution of $\left|\hat{\rho}_{a}\right|$ for a fixed $N$ and $|\rho|$ are also shown in red in Fig. 1. The distribution of $\left|\hat{\rho}_{a}\right|$ appears to have a modestly lower bias as its peak is closer to the true coherence value than the distribution of $\left|\hat{\rho}_{c}\right|$. For large values of $N$, both probability distributions tend toward $\mathbb{E}[|\rho|]$. These results hint that better estimation is possible using $\left|\hat{\rho}_{a}\right|$, however, concluding $\left|\hat{\rho}_{a}\right|$ outperforms $\left|\hat{\rho}_{c}\right|$ requires further analysis. The mean squared error for both estimators is derived below. 
D. Mean Squared Error of $\left|\hat{\rho}_{c}\right|$ and $\left|\hat{\rho}_{a}\right|$

The behavior of both coherence estimators can be examined by computing their mean squared error (MSE). The MSE of an estimator $|\hat{\rho}|$ is defined as

$$
\begin{aligned}
\operatorname{MSE}(|\hat{\rho}|) & =\mathbb{E}\left[(|\hat{\rho}|-|\rho|)^{2}\right] \\
& =\int_{0}^{1}(|\hat{\rho}|-|\rho|)^{2} p(|\hat{\rho}|) d|\hat{\rho}| .
\end{aligned}
$$

Assuming $\sigma_{f}=\sigma_{g}$ and using (10), the MSE of $\left|\hat{\rho}_{c}\right|$ can be written as

$$
\begin{aligned}
\mathbb{E}\left[\left(\left|\hat{\rho}_{c}\right|-|\rho|\right)^{2}\right]= & 2(N-1)\left(1-|\rho|^{2}\right)^{N} \int_{0}^{1}\left(\left|\hat{\rho}_{c}\right|-|\rho|\right)^{2}\left|\hat{\rho}_{c}\right|\left(1-\left|\hat{\rho}_{c}\right|^{2}\right)^{N-2}{ }_{2} F_{1}\left(N, N ; 1 ;|\rho|^{2}\left|\hat{\rho}_{c}\right|^{2}\right) d\left|\hat{\rho}_{c}\right| \\
= & 2(N-1)\left(1-|\rho|^{2}\right)^{N}\left[\int_{0}^{1}\left|\hat{\rho}_{c}\right|^{3}\left(1-\left|\hat{\rho}_{c}\right|^{2}\right)^{N-2}{ }_{2} F_{1}\left(N, N ; 1 ;|\rho|^{2}\left|\hat{\rho}_{c}\right|^{2}\right) d\left|\hat{\rho}_{c}\right|\right. \\
& -\int_{0}^{1} 2|\rho|\left|\hat{\rho}_{c}\right|^{2}\left(1-\left|\hat{\rho}_{c}\right|^{2}\right)^{N-2}{ }_{2} F_{1}\left(N, N ; 1 ;|\rho|^{2}\left|\hat{\rho}_{c}\right|^{2}\right) d\left|\hat{\rho}_{c}\right| \\
& \left.+\int_{0}^{1}|\rho|^{2}\left|\hat{\rho}_{c}\right|\left(1-\left|\hat{\rho}_{c}\right|^{2}\right)^{N-2}{ }_{2} F_{1}\left(N, N ; 1 ;|\rho|^{2}\left|\hat{\rho}_{c}\right|^{2}\right) d\left|\hat{\rho}_{c}\right|\right] .
\end{aligned}
$$

Following [17],

$$
\int_{0}^{1}(1-x)^{\mu-1} x_{p-1}^{\nu} F_{q}\left(a_{1}, \ldots, a_{p} ; b_{1}, \ldots, b_{q} ; a x\right) d x=\frac{\Gamma(\mu) \Gamma(\nu)}{\Gamma(\mu+\nu)}{ }_{p+1} F_{q+1}\left(\nu, a_{1}, \ldots, a_{p} ; \mu+\nu, b_{1}, \ldots, b_{q} ; a\right)
$$

where $\Gamma(\cdot)$ is the Gamma function and ${ }_{p} F_{q}(\cdot, \cdot ; \cdot ; \cdot)$ is the generalized hypergeometric function, the analytical expression for the MSE of $\left|\hat{\rho}_{c}\right|$ is

$$
\begin{aligned}
\mathbb{E}\left[\left(\left|\hat{\rho}_{c}\right|-|\rho|\right)^{2}\right]= & \Gamma(N)\left(1-|\rho|^{2}\right)^{N} \\
& \cdot\left[\frac{\Gamma(2)}{\Gamma(N+1)}{ }_{3} F_{2}\left(2, N, N ; N+1,1 ;|\rho|^{2}\right)-2|\rho| \frac{\Gamma\left(\frac{3}{2}\right)}{\Gamma\left(N+\frac{1}{2}\right)}\right. \\
& \cdot{ }_{3} F_{2}\left(\frac{3}{2}, N, N ; N+\frac{1}{2}, 1 ;|\rho|^{2}\right) \\
& \left.+|\rho|^{2} \frac{1}{\Gamma(N)}{ }_{3} F_{2}\left(1, N, N ; N, 1 ;|\rho|^{2}\right)\right] .
\end{aligned}
$$


Similarly, the MSE of $\left|\hat{\rho}_{a}\right|$ is

$$
\begin{aligned}
\mathbb{E}\left[\left(\left|\hat{\rho}_{a}\right|-|\rho|\right)^{2}\right]= & \Gamma\left(N+\frac{1}{2}\right)\left(1-|\rho|^{2}\right)^{N} \\
& \cdot\left[\frac{\Gamma(2)}{\Gamma\left(N+\frac{3}{2}\right)}{ }_{3} F_{2}\left(2, N, N+\frac{1}{2} ; N+\frac{3}{2}, 1 ;|\rho|^{2}\right)-2|\rho| \frac{\Gamma\left(\frac{3}{2}\right)}{\Gamma(N+1)}\right. \\
& \cdot{ }_{3} F_{2}\left(\frac{3}{2}, N, N+\frac{1}{2} ; N+1,1 ;|\rho|^{2}\right) \\
+ & \left.|\rho|^{2} \frac{1}{\Gamma\left(N+\frac{1}{2}\right)}{ }_{3} F_{2}\left(1, N, N+\frac{1}{2} ; N+\frac{1}{2}, 1 ;|\rho|^{2}\right)\right] .
\end{aligned}
$$

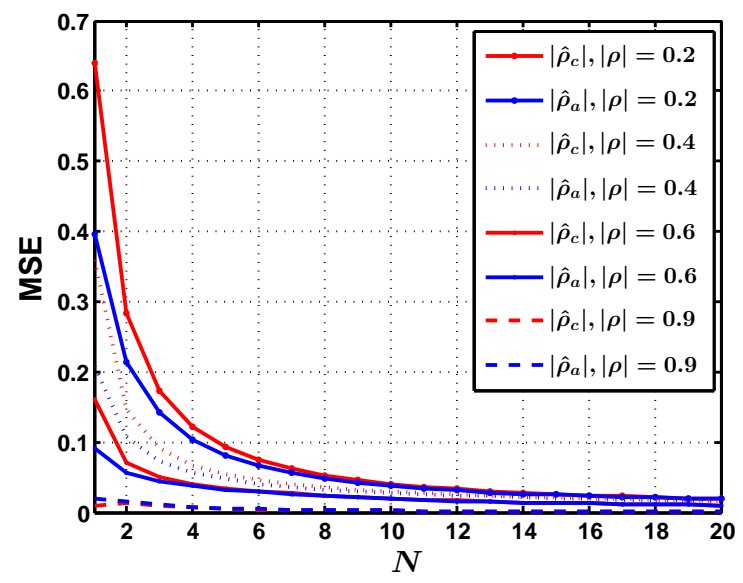

(a)

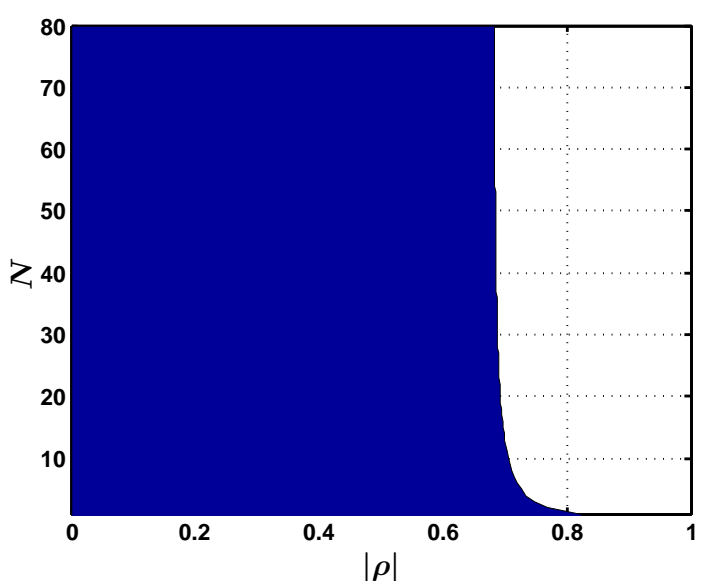

(b)

Fig. 2: (a) Mean squared error versus $N$ for $|\rho|=0.2,|\rho|=0.4,|\rho|=0.6$ and $|\rho|=0.9$. (b) The blue region indicates the domain where $\operatorname{MSE}\left(\left|\hat{\rho}_{a}\right|\right)<\operatorname{MSE}\left(\left|\hat{\rho}_{c}\right|\right)$ in a range of $N$ from 1 to 80.

Fig. 2 (a) plots the MSE values with respect to the number of samples for true coherence values of $|\rho|=0.2,|\rho|=0.4,|\rho|=0.6$, and $|\rho|=0.9$. Note that the MSE values of $\left|\hat{\rho}_{a}\right|$ are typically less than the MSE values of $\left|\hat{\rho}_{c}\right|$, especially when $|\rho|$ is low, i.e., change has occurred. In Fig. 2 (b), the blue surface is the region where $\operatorname{MSE}\left(\left|\hat{\rho}_{a}\right|\right)<\operatorname{MSE}\left(\left|\hat{\rho}_{c}\right|\right)$. Note that $\operatorname{MSE}\left(\left|\hat{\rho}_{a}\right|\right)<\operatorname{MSE}\left(\left|\hat{\rho}_{c}\right|\right)$ when the coherence is low, especially when a small number of samples is used for estimation. For large values of $N$, the changeover point is $|\rho| \approx .7$.

From this MSE analysis, we can conclude that $\left|\hat{\rho}_{a}\right|$ is better than $\left|\hat{\rho}_{c}\right|$ for change detection at least when $\sigma_{f} \approx \sigma_{g}$ (a condition for which a first stage detector can screen) and $|\rho| \lesssim 0.7$. Change, 
the state of interest in change detection, is represented by $\rho \approx 0$, where $\operatorname{MSE}\left(\left|\hat{\rho}_{a}\right|\right)<\operatorname{MSE}\left(\left|\hat{\rho}_{c}\right|\right)$. Second, the size of $N$ is limited in practice in $\operatorname{SAR~CCD,~which~leads~to~} \operatorname{MSE}\left(\left|\hat{\rho}_{a}\right|\right)<\operatorname{MSE}\left(\left|\hat{\rho}_{c}\right|\right)$ for more values of $|\rho|$. Finally, change detection error is not equal to coherence estimation error. Note from Fig. 2 that the magnitude of the difference in estimation errors is small for large values of $|\rho|$, where $\operatorname{MSE}\left(\left|\hat{\rho}_{a}\right|\right)>\operatorname{MSE}\left(\left|\hat{\rho}_{c}\right|\right)$. For other values of $|\rho|$, especially small values, i.e., $|\rho|=.2$, the MSE for $\left|\hat{\rho}_{a}\right|$ is more than two tenths smaller than the MSE for $\left|\hat{\rho}_{c}\right|$. The MSE analysis is valid when the assumption that $\sigma_{f}=\sigma_{g}$ is met. Therefore, a two-stage change detection approach that applies intensity change detection so that only samples such that $\sigma_{f} \approx \sigma_{g}$ are passed to the second stage $\left|\hat{\rho}_{a}\right|$, is considered in detail in the next section.

\section{Two-StAGe Change Detection}

Coherence and intensity ratio statistics are used separately to detect change at different scales, but if change detection, regardless of scale, is the goal, these statistics should be combined to reveal change at all scales. Combining the statistics has the additional benefit of testing first for equal variance, justifying the assumption of equal variance in order to use a more accurate coherence estimator; specifically, the second step using the alternative Berger coherence estimator $\hat{\rho}_{a}$ is proposed. The combined test therefore is expected to provides better change detection than either statistic alone. The procedure for this proposed test is illustrated in Fig. 3.

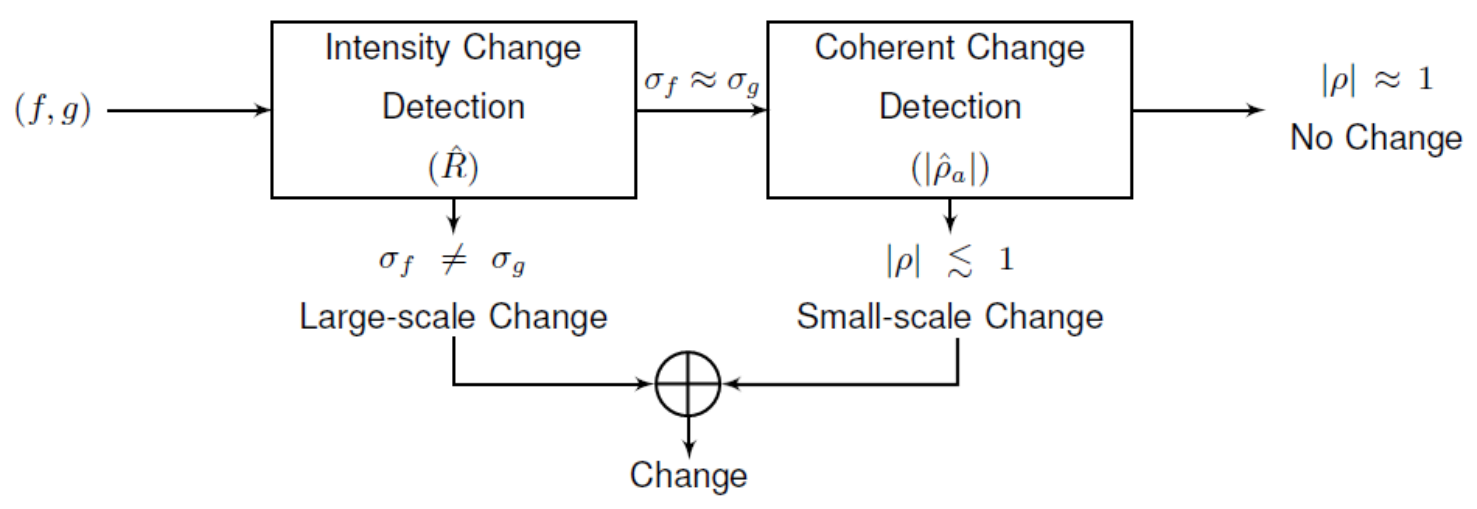

Fig. 3: The block diagram of the two-stage change detection scheme

In order to detect change pixels at all scales, we first apply the intensity change detector $\hat{R}$ that identifies pixel pairs with large variance changes, i.e. $\sigma_{f} \neq \sigma_{g}$ such that good SAR change 
detection image contrast is achieved. The pixel pairs that are identified to have equal or near equal variances in the first stage are used as an input to the second stage. This leads to most pairs entering the second stage having nearly equal variance. The second stage test uses the alternative coherence estimator $\left|\hat{\rho}_{a}\right|$ to detect subtle changes. The output of the first and the second stages, large-scale change and small-scale change, respectively, are combined to form the final change detection result.

It is shown in Appendix that for arbitrary positive values of $\sigma_{f}, \sigma_{g},|\rho| \leq 1$, and $N$, the exact joint probability density function of $\left|\hat{\rho}_{a}\right|$ and $\hat{R}$ is given by

$$
\begin{aligned}
p\left(\left|\hat{\rho}_{a}\right|, \hat{R}\right)= & \frac{\left(1-|\rho|^{2}\right)^{N} \Gamma(2 N)}{\Gamma(N) \Gamma(N-1)} \frac{\left|\hat{\rho}_{a}\right|}{2(\hat{R}+1)^{2}}\left[\frac{1}{\hat{R}+1}\left(1-\frac{1}{\hat{R}+1}\right)-\frac{\left|\hat{\rho}_{a}\right|^{2}}{4}\right]^{N-2} \\
& \cdot\left[\left|\hat{\rho}_{a}\right||\rho|+\frac{\sigma_{g}^{2} \hat{R}+\sigma_{f}^{2}}{(\hat{R}+1) \sigma_{f} \sigma_{g}}\right]^{-2 N} F_{1}\left(\frac{1}{2}, 2 N, 1, \frac{2\left|\hat{\rho}_{a}\right||\rho|}{\left|\hat{\rho}_{a}\right||\rho|+\frac{\sigma_{g}^{2} \hat{R}+\sigma_{f}^{2}}{(\hat{R}+1) \sigma_{f} \sigma_{g}}}\right)
\end{aligned}
$$

subject to

$$
\left|\hat{\rho}_{a}\right| \leq\left[4 \hat{R} /(\hat{R}+1)^{2}\right]^{\frac{1}{2}}, 0 \leq \hat{R}<\infty
$$

Examples of $p\left(\left|\hat{\rho}_{a}\right|, \hat{R}\right)$ for fixed $\sigma_{f}, \sigma_{g},|\rho|$, and $N$ are shown in Fig. 4, (a) with $|\rho|=0.1$ and $R=0.1\left(\sigma_{f}=0.3015\right.$ and $\left.\sigma_{g}=0.9535\right)$, (b) with $|\rho|=0.4$ and $R=0.76\left(\sigma_{f}=0.6571\right.$ and $\left.\sigma_{g}=0.7538\right)$, and (c) with $|\rho|=0.9$ and $R=0.9\left(\sigma_{f}=0.6882\right.$ and $\left.\sigma_{g}=0.7255\right)$ for $N=3$. From these figures, we see that $p\left(\left|\hat{\rho}_{a}\right|, \hat{R}\right)$ is only non-zero in the domain where (18) holds.

We test the constraint in (18) by plotting the 2-D histogram of the SAR data shown in Fig. 5 based on $\hat{R}$ and $\left|\hat{\rho}_{a}\right|$ in Fig. 6 (a). Parameters from Fig. 6 (a) are estimated by the maximum likelihood estimator and the estimated parameters $\left(\hat{R}=0.8485\right.$ and $\left.\left|\hat{\rho}_{a}\right|=0.9899\right)$ are used as the true parameters of $p\left(\left|\hat{\rho}_{a}\right|, \hat{R}\right)$ in Fig. 6 (b). Notice no data points of the histogram falls outside of the theoretical boundary of the pdf. With $p\left(\left|\hat{\rho}_{a}\right|, \hat{R}\right)$, the probability of detection and the probability of false alarm as a function of detection thresholds in each stage can be numerically computed.

\section{Heuristic Approach to Two-Stage Change Detection}

The focus of this initial study is the development of a heuristic approach to choosing thresholds due to the integral of (17) being non-trivial to be solved analytically. A detailed study using 


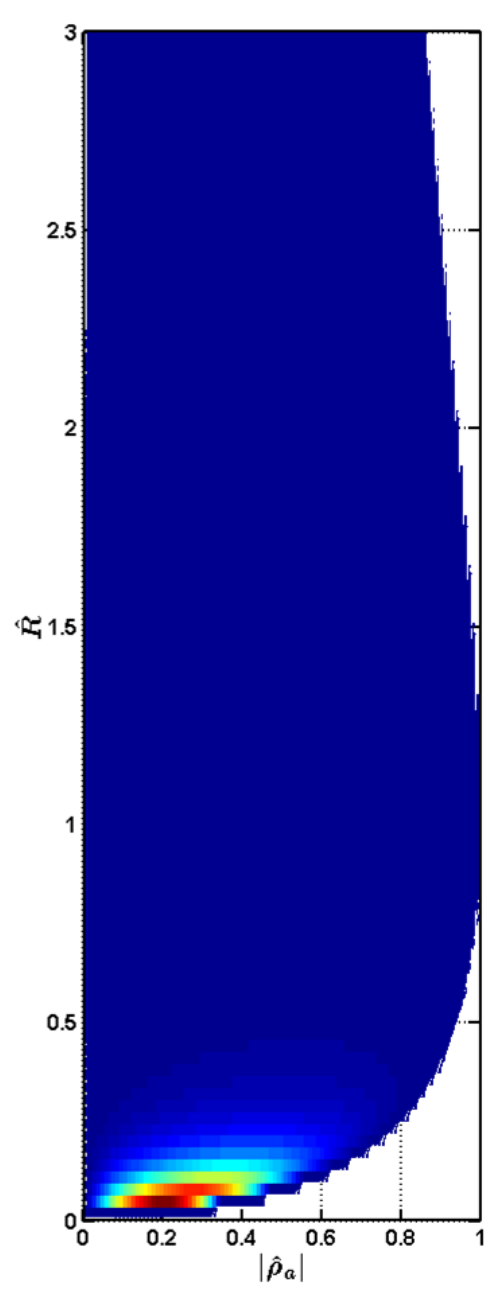

(a)

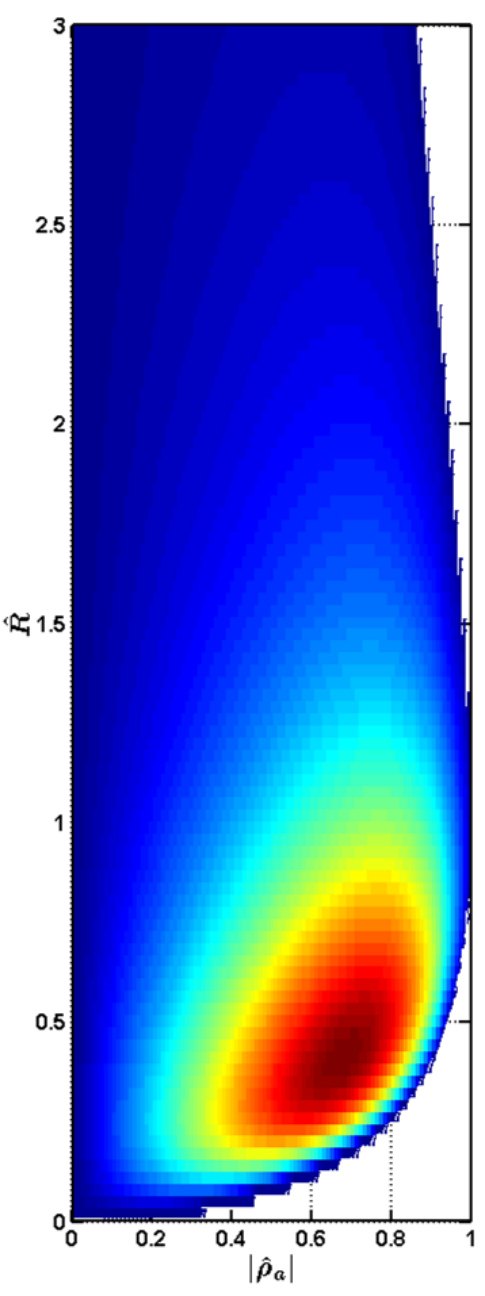

(b)

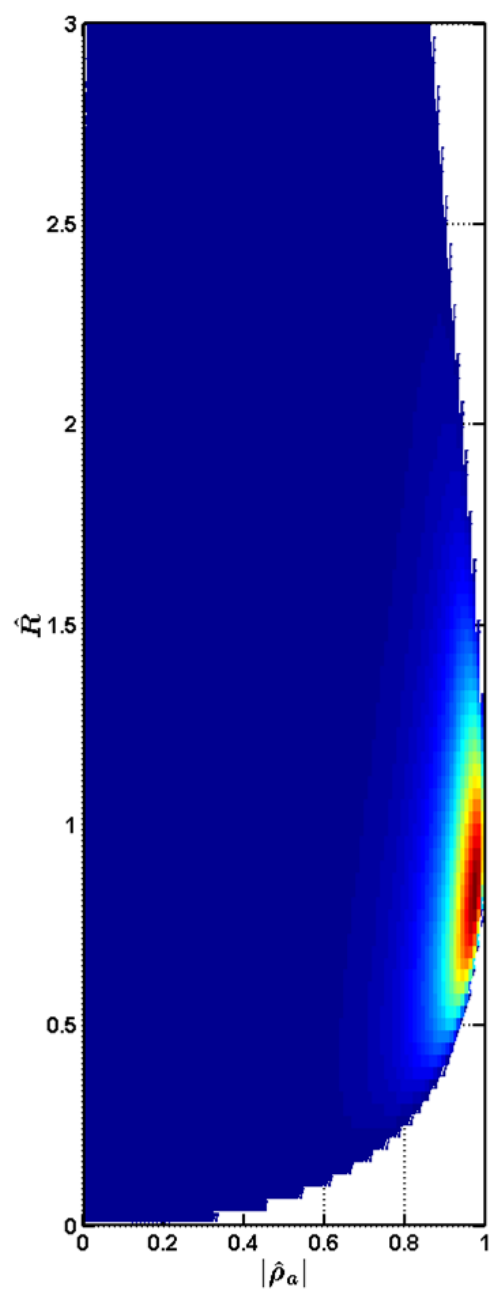

(c)

Fig. 4: Example figures for $p\left(\left|\hat{\rho}_{a}\right|, \hat{R}\right)$ when (a) $R=0.1$ and $|\rho|=0.1$, (b) $R=0.75$ and $|\rho|=0.4$, and (c) $R=0.9$ and $|\rho|=0.9$ for $N=3$

(17) to explore the inherent behavior of the two-stage change detector will be presented in the future work.

\section{A. Analysis of SAR}

The two-stage change detection can be described as two sequential hypothesis tests involving data-based estimates of $\sigma_{f}$ and $\sigma_{g}$ in the first stage, and $|\rho|$ in the second stage. We can write 

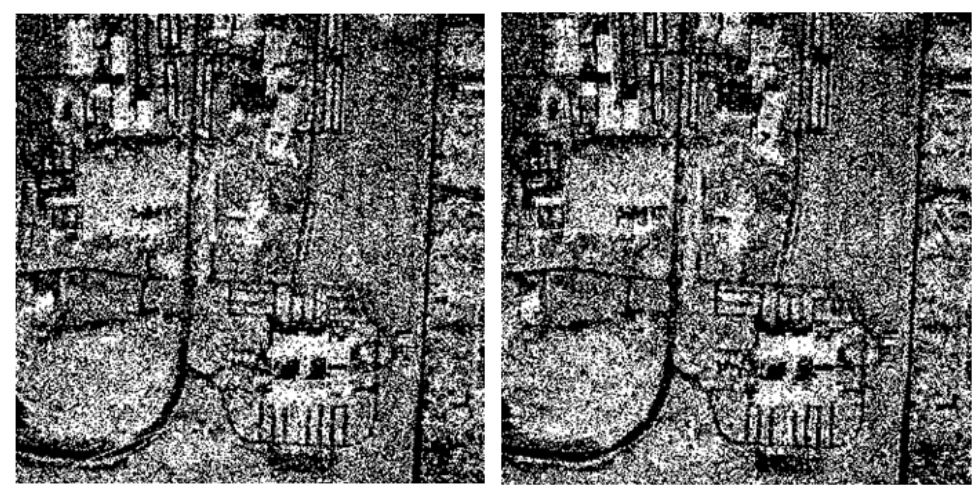

Fig. 5: $4501 \times 4501$ SAR magnitude image pair, available publicly from the Air Force Research Laboratory SAR data set described in [18].

TABLE I: Final hypothesis tests of the two-stage change detection

\begin{tabular}{|l|c|c|}
\hline & $\sigma_{f} \approx \sigma_{g}$ & $\sigma_{f} \neq \sigma_{g}$ \\
\hline$|\rho| \approx 1$ & $H_{0}$ & $H_{1}$ \\
\hline$|\rho| \lesssim 1$ & $H_{1}$ & $H_{1}$ \\
\hline
\end{tabular}

the hypothesis tests as

$$
\begin{array}{ll}
H_{0}^{1 s t}: \quad \sigma_{f} \approx \sigma_{g} \quad H_{1}^{1 s t}: \quad \sigma_{f} \neq \sigma_{g} \\
H_{0}^{2 n d}: \quad|\rho| \approx 1 \quad H_{1}^{2 n d}: \quad|\rho| \lesssim 1 .
\end{array}
$$

The null hypothesis of the first stage test is that the population variances are equal or near equal and the alternative hypothesis is that those populations have different variances. The null hypothesis in the second stage test is that $|\rho| \approx 1$ and the alternative hypothesis is that $|\rho| \lesssim 1$. Given the two sets of hypothesis tests, we can form the final hypothesis tests of the two-stage change detector as shown in Table I. Fig. 7 (b) illustrates the dichotomy of the final hypothesis tests, $H_{0}$ (no change) and $H_{1}$ (change) overlayed on top of the domain $\hat{R} \leq 3$ where $p\left(\left|\hat{\rho}_{a}\right|, \hat{R}\right)$ is non-zero.

In the first stage, $\sigma_{f} \neq \sigma_{g}$ constitutes change, more specifically a large-scale change as labeled in Fig. 3. The sample pairs that are identified to have $\sigma_{f} \approx \sigma_{g}$ in the first stage are passed to the second stage. In the second stage, the sample pairs with $\sigma_{f} \approx \sigma_{g}$ and $|\rho| \lesssim 1$ represents change ('Small-scale Change' in Fig. 3) and $\sigma_{f} \approx \sigma_{g}$ and $|\rho| \approx 1$ represents no change ('No Change' in Fig. 3). The small-scale change and the large-scale change are combined to form the final 


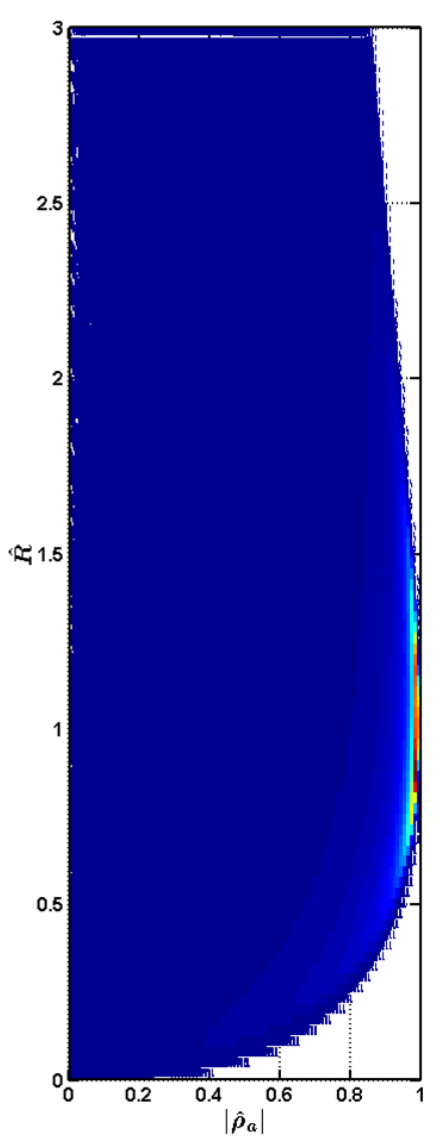

(a)

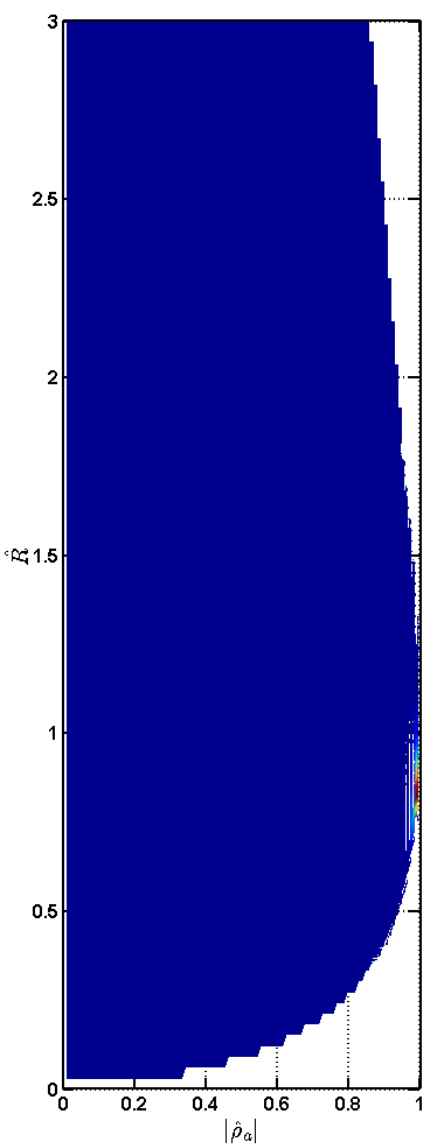

(b)

Fig. 6: (a) 2-D histogram of SAR in Fig. 5 in $\hat{R}$ and $\left|\hat{\rho}_{a}\right|$ for $\hat{R} \leq 3$ (b) $p\left(\left|\hat{\rho}_{a}\right|, \hat{R}\right)$ when the estimated $R$ and $|\rho|$ over the entire image are used as the true parameter $(R=0.8485$ and $|\rho|=0.9899)$.

change $H_{1}$ and the no change output from the second stage is used to form the final no change $H_{0}$.

Among the two sets of hypothesis tests $H^{1 s t}$ and $H^{2 n d}$, one can form four possible outcomes: $H_{0}^{1 s t}+H_{0}^{2 n d}, H_{0}^{1 s t}+H_{1}^{2 n d}, H_{1}^{1 s t}+H_{0}^{2 n d}$, and $H_{1}^{1 s t}+H_{1}^{2 n d}$. As shown in Fig. 7 (a), we notice $H_{0}^{1 s t}+H_{0}^{2 n d}$ indicates the final no change $H_{0}$ and $H_{0}^{1 s t}+H_{1}^{2 n d}$ represents a subset of the final change $H_{1}$. We also notice both $H_{1}^{1 s t}+H_{1}^{2 n d}$ and $H_{1}^{1 s t}+H_{0}^{2 n d}$ represent a subset of $H_{1}$, however, we expect that it is unlikely to observe sample pairs under $H_{1}^{1 s t}+H_{0}^{2 n d}$. We can identify that $H_{1}^{1 s t}+H_{0}^{2 n d}$ is unlikely based on intuition because any large-scale change will affect phase, 


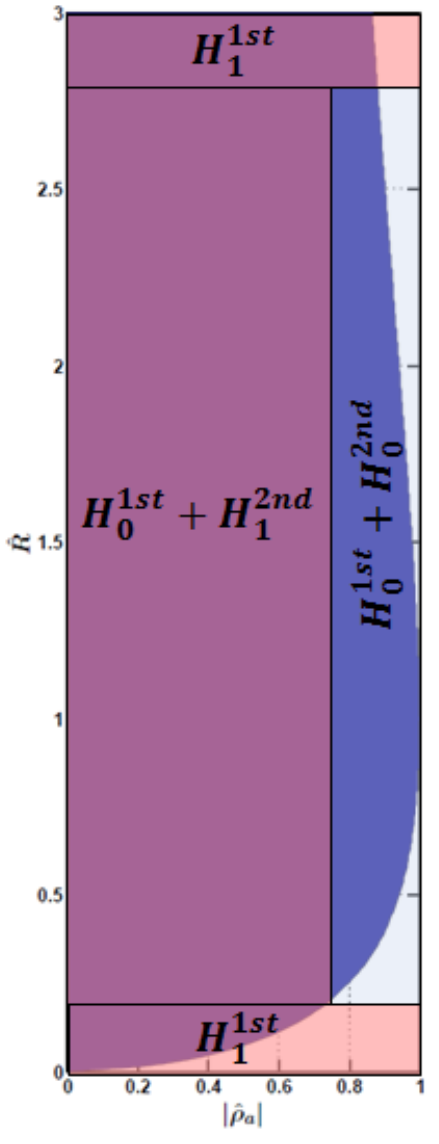

(a)

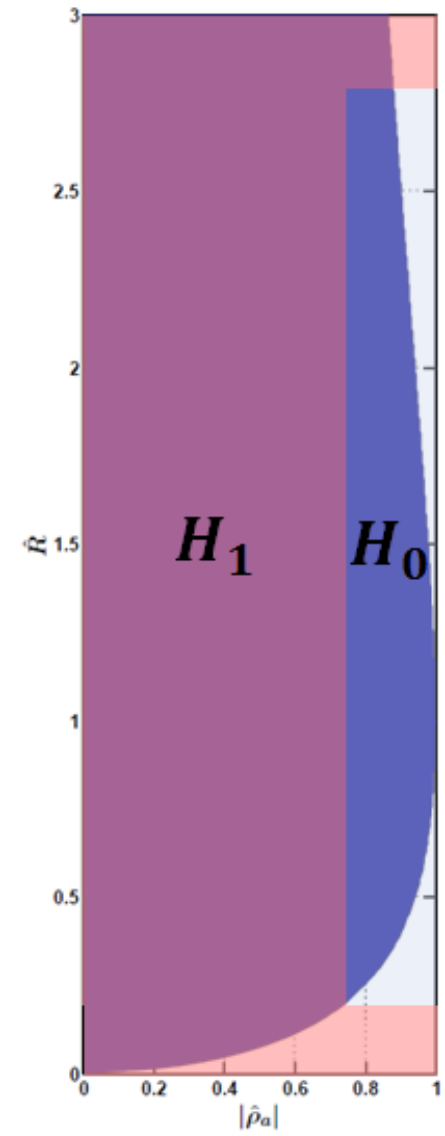

(b)

Fig. 7: Dichotomy of (a) the first and the second stage change detection outputs (b) the final change $H_{1}$ and the final no change $H_{0}$, overlayed on top of the domain $\hat{R} \leq 3$ where $p\left(\left|\hat{\rho}_{a}\right|, \hat{R}\right)$ is non-zero.

leading to small-scale changes as well. The unlikely outcome can be seen theoretically via the constraint of $p\left(\left|\hat{\rho}_{a}\right|, \hat{R}\right)$ in (18). The constraint implies $\left|\hat{\rho}_{a}\right| \lesssim 2 \sqrt{\epsilon}$ for $\hat{R} \approx \epsilon>0$, where $\epsilon$ is a small positive infinitesimal quantity. This also implies high $|\rho|$ is unlikely to occur with $R$ far from unity. Fig. 7 (b) illustrates this fact by showing no data points underneath where $H_{1}^{1 s t}+H_{0}^{2 n d}$ holds. As there is an interplay between the first and the second stage tests, in the next section, we discuss our methods of determining thresholds for each stage of the two-stage change detection scheme. 
B. Threshold Selection for $\hat{R}$

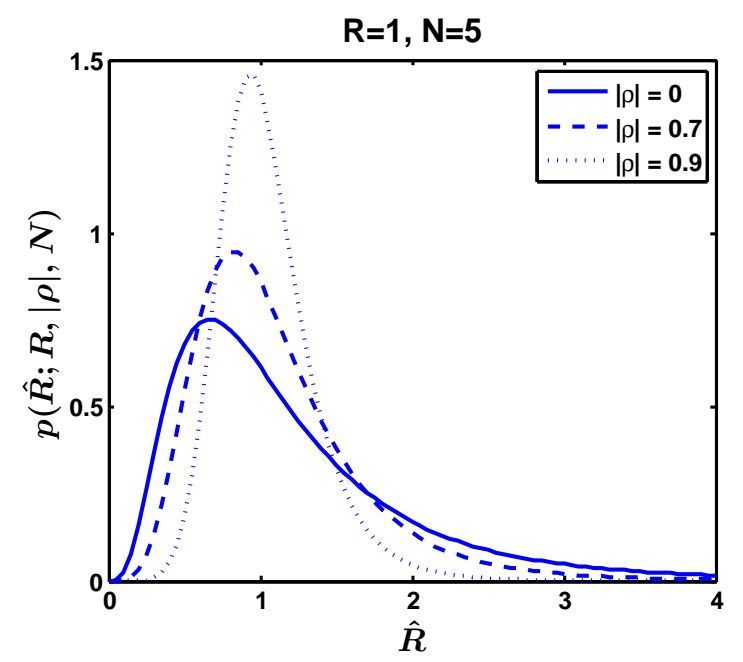

Fig. 8: Probability density function plot of $\hat{R}$ in (6) shown as a function of $|\rho|$ when $H_{0}^{1 s t}$ is true and $N=5$

Thresholds for a detection problem are traditionally determined assuming the null hypothesis is true. Similarly, we determine the thresholds $R_{l, \alpha}$ and $R_{u, \alpha}$ of the first stage test, assuming $H_{0}^{1 s t}$ is true, i.e. $\sigma_{f} \approx \sigma_{g}$. Note, however, that in practice the true parameter $|\rho|$ is unknown for selection of thresholds to guarantee an acceptance level of $\alpha$. Exploring the effects of correlation parameter $|\rho|$ on the pdf of $\hat{R}$ under $H_{0}^{1 s t}$, Fig. 8 shows that using any value of correlation parameter such that $|\rho| \neq 0$ only decreases the variance of $\hat{R}$. That is, if we were to select thresholds of $\hat{R}$ under $H_{0}\left(\sigma_{f} \approx \sigma_{g}\right.$ and $\left.|\rho| \approx 1\right)$ for arbitrary $|\rho|$, the test is likely to reject near equal variance populations from entering the second stage test more often than expected. Moreover, analysis with real data suggests that choosing threshold values for $\hat{R}$ under the $\rho=0$ assumption for $\alpha=0.01$ captures prominent intensity changes between SAR images without introducing significant false alarms. Thus, $R_{l, \alpha}$ and $R_{u, \alpha}$ will be chosen according to (8) so that samples declared as change in the first stage indicate prominent intensity change, and therefore immediately declared as $H_{1}$. The ultimate effect of using $\hat{R}$ for change detection is that more samples with $\sigma_{f} \neq \sigma_{g}$ pass to the second stage. We discuss this event in more detail in the next subsection. 


\section{C. $\left|\hat{\rho}_{a}\right|$ with $\sigma_{f} \neq \sigma_{g}$}

The alternative coherence estimator $\left|\hat{\rho}_{a}\right|$ is employed under the assumption that underlying variances of two populations are equal $\left(\sigma_{f}=\sigma_{g}\right)$. When the equal variance assumption is met, we have shown that $\left|\hat{\rho}_{a}\right|$ has more desirable change detection properties than $\left|\hat{\rho}_{c}\right|$ (see Section II-D); when $|\rho|$ is low, $\left|\hat{\rho}_{a}\right|$ yields significantly less mean squared error than $\left|\hat{\rho}_{c}\right|$, and when $|\rho|$ is high, $\left|\hat{\rho}_{a}\right|$ yields slightly more error than $\left|\hat{\rho}_{c}\right|$, but the magnitude of the difference in estimation error is negligible. Therefore, it is expected that the more accurate estimation of small values of $|\rho|$ makes detecting true change more likely, and outweighs the slight increase in false alarms that corresponds to high values of $|\rho|$.

The behavior of $\left|\hat{\rho}_{a}\right|$ when $\sigma_{f} \neq \sigma_{g}$ can be explained with a direct comparison of distributional properties. Based on properties of inequality of arithmetic and geometric means, $\left|\hat{\rho}_{a}\right| \leq\left|\hat{\rho}_{c}\right|$ with equality if and only if $A_{11}=A_{22}$. In other words, the further $R$ gets from unity, the lower $\left|\hat{\rho}_{a}\right|$ will become compared to $\left|\hat{\rho}_{c}\right|$. This leads to more likely detection of true change when $|\rho|$ is low, and conversely increasing false alarms when $|\rho|$ is high. However, we know from (18) that $\left|\hat{\rho}_{a}\right| \leq\left[4 \hat{R} /(\hat{R}+1)^{2}\right]^{\frac{1}{2}}$ must hold. This constraint implies high $|\rho|$ is unlikely to occur with $R$ far from one, which would be the case that would cause false alarms. Therefore, a significant improvement in change detection is expected to be observed in using $\left|\hat{\rho}_{a}\right|$ compared to using $\left|\hat{\rho}_{c}\right|$, even when the equal variance assumption is not met.

\section{EXPERIMENTAL RESUlTS}

\section{A. Simulation}

We performed a simulation with known ground truth to compare the change detection performance of $\left|\hat{\rho}_{a}\right|,\left|\hat{\rho}_{c}\right|$, and the two-stage framework. For the purpose of this experiment, $|\rho|=0$ with a range of values in $R$ was chosen to indicate change, and $|\rho|=0.9$ with $R=0.9$ to indicate no change. To restrict the unlikely case, the variance ratio $R$ was fixed in generating 'no change' samples, and only varied in producing 'change' samples. Note that choosing $|\rho|=1$ or $R=1$ would result in no variability between samples, which is not realistic in SAR data. Coherence is affected by factors other than scene change, making $|\rho|=0.9$ a reasonably high coherence value. Results in detecting change corresponding to $|\rho|=0$ with a range of values in $R$ versus $|\rho|=0.9$ with $R=0.9$ were obtained using $10^{5}$ independent Monte Carlo trials, 
for sample sizes $N=3$ and $N=6$. As discussed in Section IV-B, thresholds for the first stage test are fixed to have a $99 \%$ acceptance rate $(\alpha=0.01)$ under $\sigma_{f}=\sigma_{g}$ and $|\rho|=0$ to avoid significant false alarms occurred in the first stage. Threshold for the second stage test is empirically determined through receiver operating characteristic (ROC) curve analysis. In the following analysis, ROC curves are generated varying thresholds of the second stage test after fixing thresholds for the first stage test according to Section IV-B.

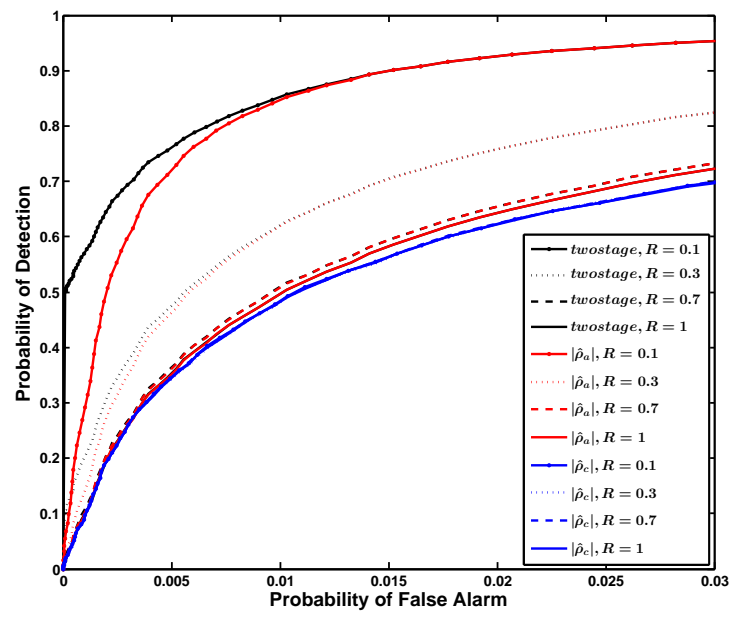

(a)

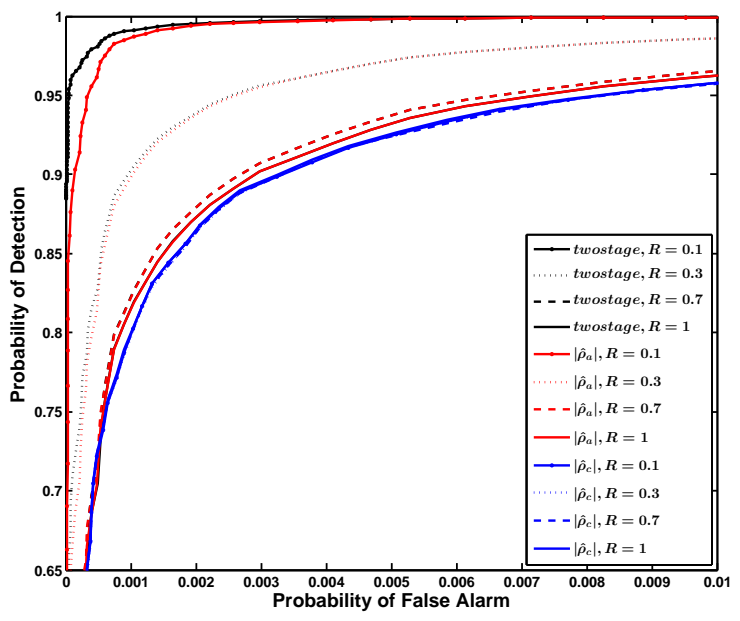

(b)

Fig. 9: Simulated ROC curves comparing the performance of $\left|\hat{\rho}_{a}\right|,\left|\hat{\rho}_{c}\right|$, and $\left|\hat{\rho}_{a}\right|$ within the twostage framework for variance ratios of $0.1,0.3,0.7$, and 1 , with sample sizes of (a) $N=3$ and (b) $N=6$.

Figs. 9 (a) and (b) show the ROC curves for the change detection methods with varying variance ratios $R$, with sample sizes of $N=3$ and $N=6$, respectively. The two-stage method is plotted in black, the classical coherence magnitude estimator $\left|\hat{\rho}_{c}\right|$ is denoted as a blue line, and $\left|\hat{\rho}_{a}\right|$ is represented with a red line.

First, we compare the performance of using $\left|\hat{\rho}_{c}\right|$ vs. $\left|\hat{\rho}_{a}\right|$. Unlike $\left|\hat{\rho}_{a}\right|,\left|\hat{\rho}_{c}\right|$ is invariant to $\sigma_{f}$ and $\sigma_{g}$, therefore the curves for $\left|\hat{\rho}_{c}\right|$ are unaffected by the different values of $R$. Our empirical studies indicate that change detection with $\left|\hat{\rho}_{a}\right|$ performs better than change detection using $\left|\hat{\rho}_{c}\right|$, not only when the true underlying variances are equal $(R=1)$, but also when they are far apart. As discussed in Subsection IV-C, the performance of change detection using $\left|\hat{\rho}_{a}\right|$ increases as 
$R$ gets further away from one, meaning a deviation from underlying assumptions unexpectedly leads to an improvement in change detection. Recall that change detection error is not the same as coherence estimation error. Fig. 9 (a) shows that the change detection with $\left|\hat{\rho}_{a}\right|$ can achieve a nearly $37 \%$ increase in probability of detection $\left(\mathrm{P}_{\mathrm{D}}\right)$ at a $1 \%$ probability of false alarm $\left(\mathrm{P}_{\mathrm{FA}}\right)$ compared to using $\left|\hat{\rho}_{c}\right|$ when $R=0.1$.

Next, we compare the change detection performance using the two-stage method against using only $\left|\hat{\rho}_{a}\right|$. Since the two-stage method uses the intensity change detector in the first stage, and $\left|\hat{\rho}_{a}\right|$ in the second stage, the two-stage method curve converges to $\left|\hat{\rho}_{a}\right|$. The performance improvement of the two-stage method compared to $\left|\hat{\rho}_{a}\right|$ is especially prominent at low $\mathrm{P}_{\mathrm{FA}}$ and low $R$. The additional intensity change detection step allows the two-stage method to detect the regions of low $R$ that leads to a higher $\mathrm{P}_{\mathrm{D}}$ at a limited $\mathrm{P}_{\mathrm{FA}}$ compared to other methods.

A comparison of Figs. 9 (a) and (b) confirms that overall change detection performance increases with $N$, and that, as expected with larger sample sizes, the curves tend toward the optimal operating point. However, we still observe a performance increase using the two-stage method compared to using $\left|\hat{\rho}_{a}\right|$ in Fig. 9 (b) especially at a limited $\mathrm{P}_{\mathrm{FA}}$ and low $R$. Our empirical studies indicate that with $R=0.1$ (low $R$ ), the number of samples $N=16$ yields a negligible difference $\left(<10^{-6}\right)$ in the area under the ROC curves between the two-stage method and $\left|\hat{\rho}_{a}\right|$. Similarly, the difference in the area under the curves between the two-stage method and $\left|\hat{\rho}_{c}\right|$ becomes negligible $\left(<10^{-6}\right)$ when $N=16$.

We observed the exceptional performance of the two-stage method and $\left|\hat{\rho}_{a}\right|\left(\approx 99 \% \mathrm{P}_{\mathrm{D}}\right.$ at $\left.1 \% \mathrm{P}_{\mathrm{FA}}\right)$ in Fig. 9 (b), mainly because the simulation setup does not incorporate radar processing noise and false alarms caused by uninteresting changes (radar shadow, vegetation, etc). To evaluate the performance of the change detection techniques in a more realistic setting, the following subsection applies the change detection methods to publicly available AFRL SAR data as well as SAR data collected in Yuma, AZ, in April 2008 using Boeing's Ku-band Compact Radar and a King Air 300 aircraft.

\section{B. Empirical Performance of the Two-stage Test}

As a specific example, a smaller $800 \times 400$ region of Fig. 5, shown in Figs. 10 (a) and (b) was used to compare the performance of four distinct metrics: the intensity change detector $\hat{R}$, the classical coherence estimator $\left|\hat{\rho}_{c}\right|$, the alternative coherence estimator $\left|\hat{\rho}_{a}\right|$, and the two-stage 


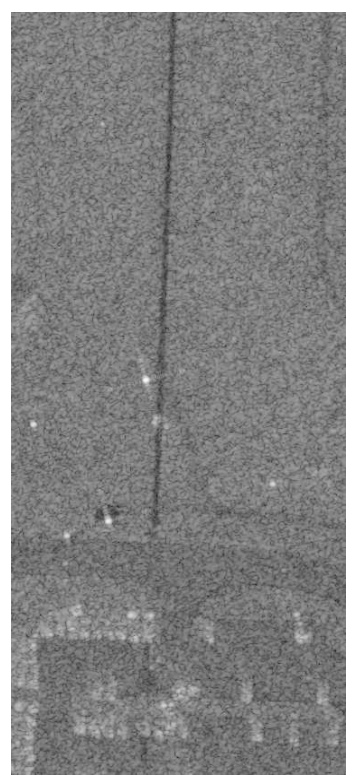

(a) Reference SAR

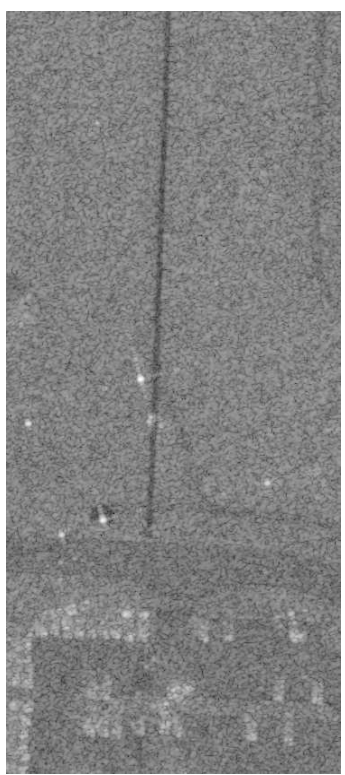

(b) Mission SAR
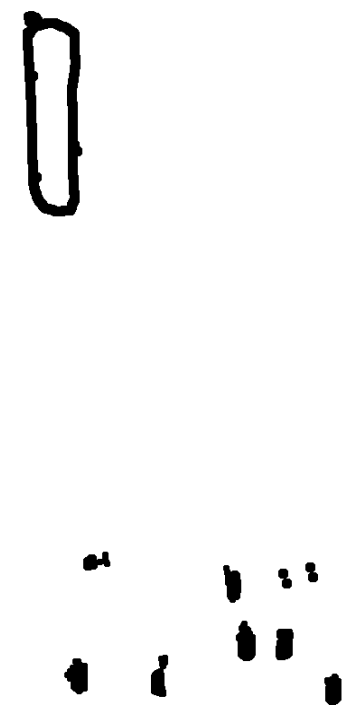

(c) Ground-truth

Fig. 10: A zoomed-in $850 \times 400$ portion of Fig. 5

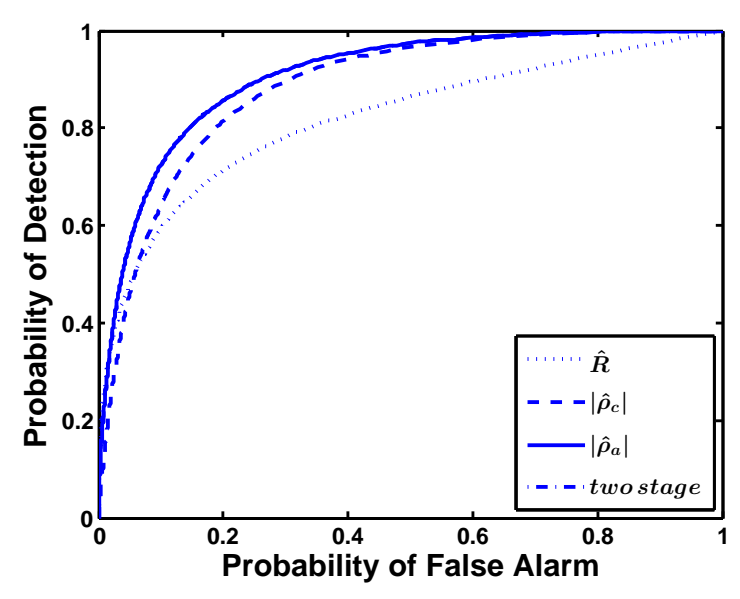

(a) Original view

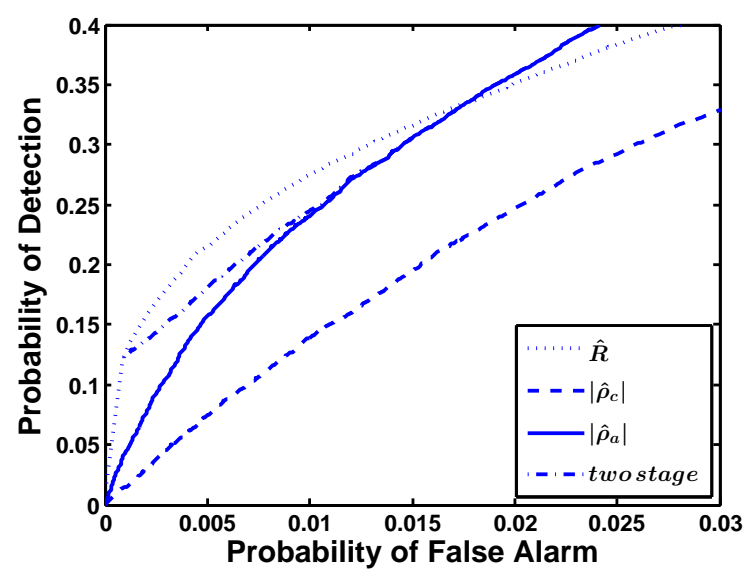

(b) A zoomed-in view of Fig. 11 (a), near $\mathrm{P}_{\mathrm{FA}} \leq 0.03$

Fig. 11: Change detection performance results on Fig. 10 using $\hat{R},\left|\hat{\rho}_{c}\right|,\left|\hat{\rho}_{a}\right|$, and two-stage test change detector. The changed and the unchanged scenes were determined by visual inspection and recorded as a binary mask as shown in Figs. 10 (c). Fig. 11 shows the performance of the four metrics on Fig. 10 (a) and (b) with $N=5$. Thresholds for the first stage test is determined 
the same way as described in Section V-A. Pixels that are declared as change in the first stage are masked as zero (a black pixel), and later combined with the raw output of $\left|\hat{\rho}_{a}\right|$. Again, ROC curves in Fig. 11 are generated varying thresholds for the second stage after fixing thresholds for the first stage test.

By comparing the two-stage test with other competing metrics in Fig. 11 (a), we notice an improvement with the two-stage method compared to $\left|\hat{\rho}_{c}\right|$. Due to the noisy nature of SAR, we are primarily interested in low $\mathrm{P}_{\mathrm{FA}}$, close to zero. Notice in Fig. 11 (b), in low $\mathrm{P}_{\mathrm{FA}}$ regions, the two-stage method performs better than $\left|\hat{\rho}_{c}\right|$ and $\left|\hat{\rho}_{a}\right|$.

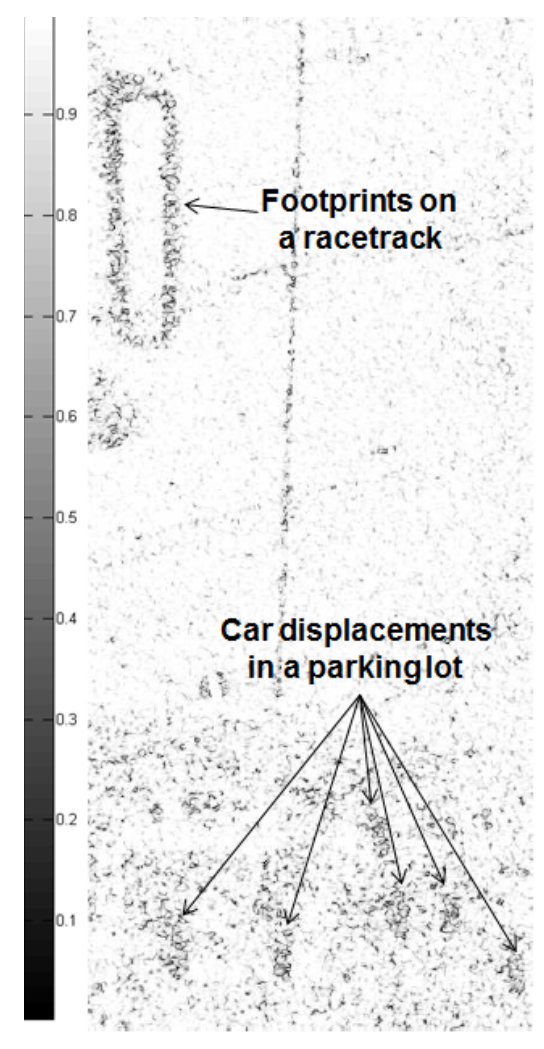

(a) $\left|\hat{\rho}_{c}\right|$ (state of the art)

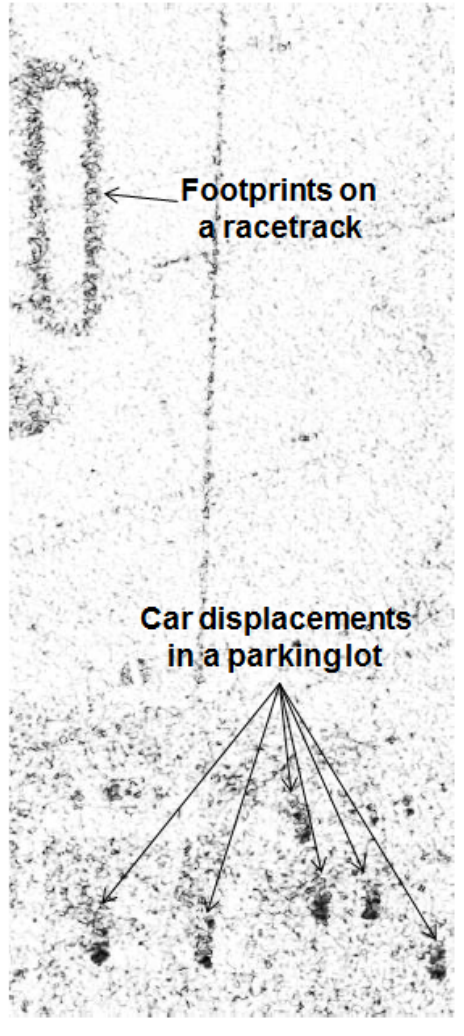

(b) $\left|\hat{\rho}_{a}\right|$

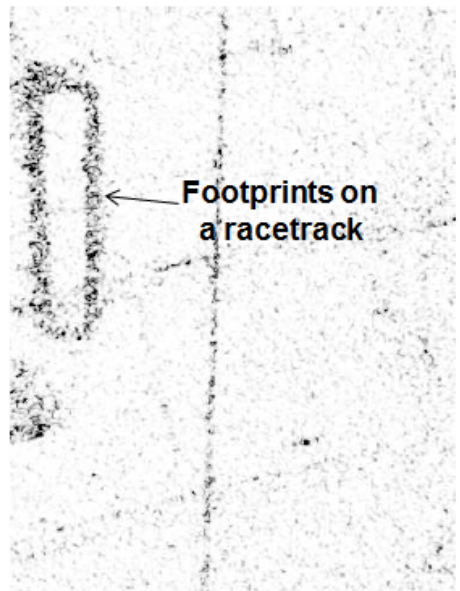

Car displacements in a parkinglot

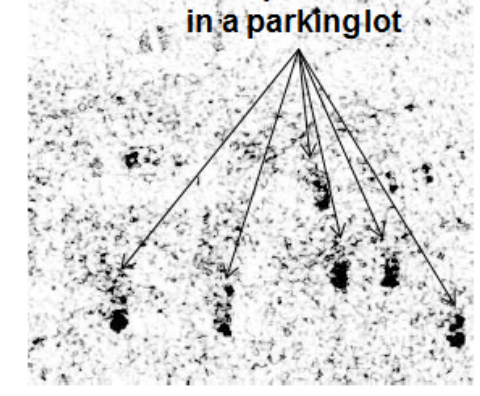

(c) Two-stage

Fig. 12: SAR change detection images formed with $N=5$, with (c) showing highest overall contrast.

In practice, raw estimator outputs ranging from 0 to 1 (e.g., images in Fig. 12) are often called SAR change detection images. As there can be numerous uninteresting changes such as vegetation and radar shadows, image analysts investigate the raw outputs and manually determine 


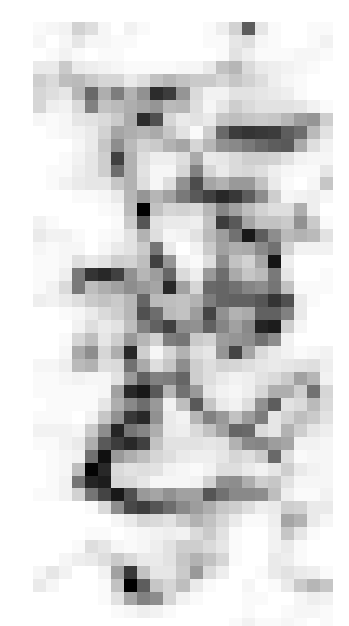

(a) $\left|\hat{\rho}_{c}\right|$ (state of the art)

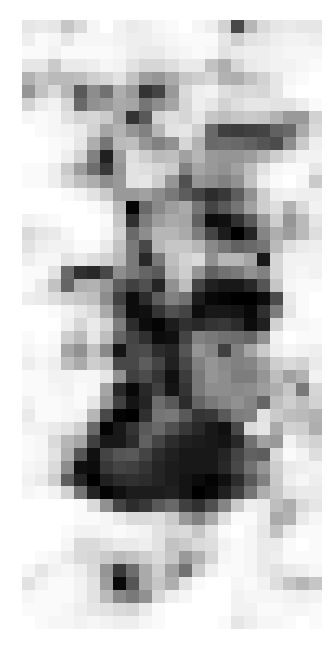

(b) $\left|\hat{\rho}_{a}\right|$

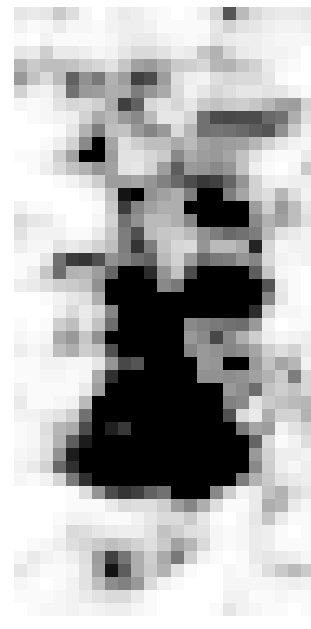

(c) Two-stage

Fig. 13: Corresponding close-up view of a car displacement in a parking lot in Fig. 12, again with (c) showing the highest contrast

regions of interesting change. In Fig. 12, we show the raw outputs of $\left|\hat{\rho}_{c}\right|,\left|\hat{\rho}_{a}\right|$, and the two-stage method, respectively, prior to thresholding. The raw output of the two-stage method is defined as the raw output of $\left|\hat{\rho}_{a}\right|$ after masking the declared change pixels with the first stage test $\hat{R}$. Note that in all three images in Fig. 12, there are considerable false alarms introduced from the ground material in the parking lot. In the first image generated with $\left|\hat{\rho}_{c}\right|$, the car displacement changes are indistinguishable from the false alarms. The second image, Fig. 12 (b) with $\left|\hat{\rho}_{a}\right|$ clearly differentiates the car displacement changes, indicated with significantly darker pixels, compared to the false alarm pixels. The third image in Fig. 12 (c) with the two-stage method further improves the result from $\left|\hat{\rho}_{a}\right|$ by emphasizing the large-scale changes.

Smaller portions of Figs. 12 are shown to further illustrate the difference between the three methods in the regions of unequal variance in Figs. 13 and nearly equal variance in Figs. 14. Fig. 13 shows a zoomed in view of a car displacement change in the parking lot from Fig. 12. Notice the center of the car displacement using $\left|\hat{\rho}_{c}\right|$ is hollow, making it difficult to identify as an object displacement change. However, the result from $\left|\hat{\rho}_{a}\right|$ fills in the hollow gap with darker pixels, indicating low coherence, and the two-stage method further enhances the result 


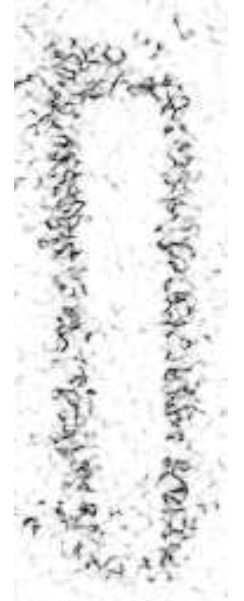

(a) $\left|\hat{\rho}_{c}\right|$ (state of the art)

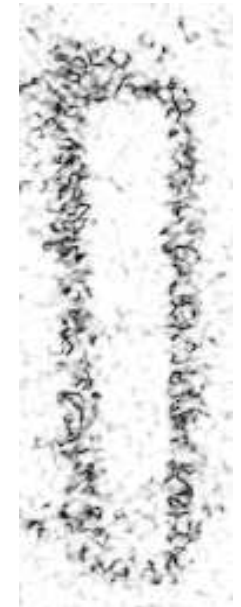

(b) $\left|\hat{\rho}_{a}\right|$

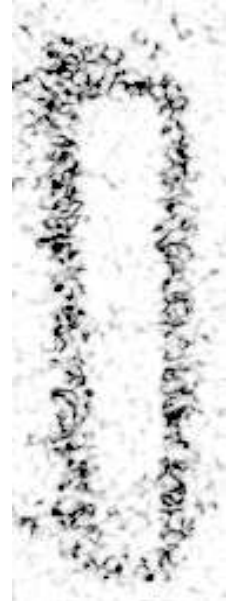

(c) Two-stage

Fig. 14: Corresponding close-up view of footprints on a race track in Fig. 12, again with (c) showing the highest contrast

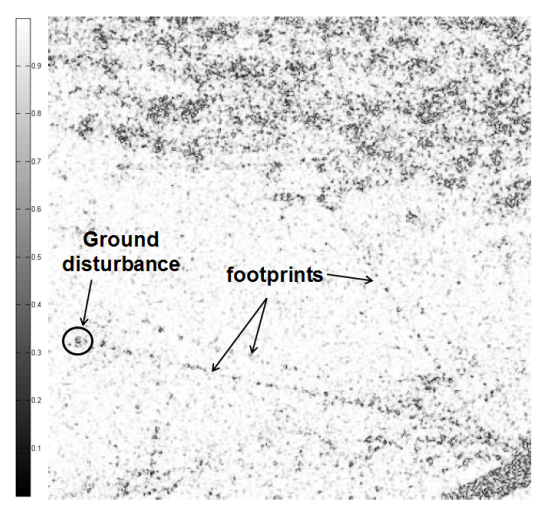

(a) $\left|\hat{\rho}_{c}\right|$ (state of the art)

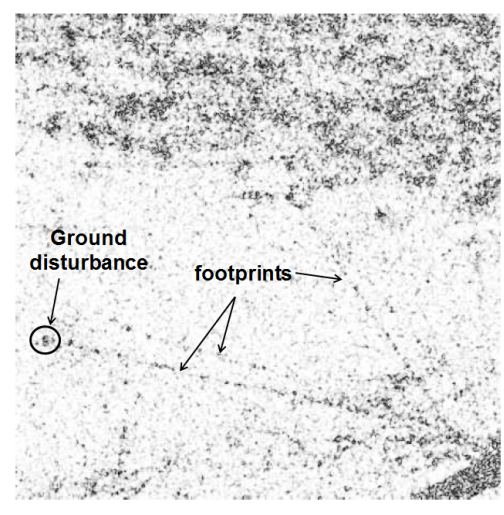

(b) $\left|\hat{\rho}_{a}\right|$

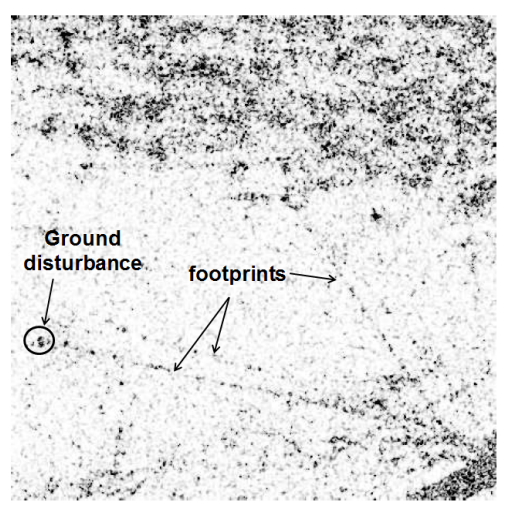

(c) Two-stage

Fig. 15: Change detection images formed using Beoing's SAR data with $N=5$

of $\left|\hat{\rho}_{a}\right|$. Fig. 14 indicates tracks. We observe a slight contrast enhancement using $\left|\hat{\rho}_{a}\right|$ or the two-stage method compared to using $\left|\hat{\rho}_{c}\right|$. In regions of both large-scale and small-scale change, the two-stage method yields the highest contrast among the three techniques.

We validate the robustness of our method on multiple SAR data. The results in Fig. 15 shows 


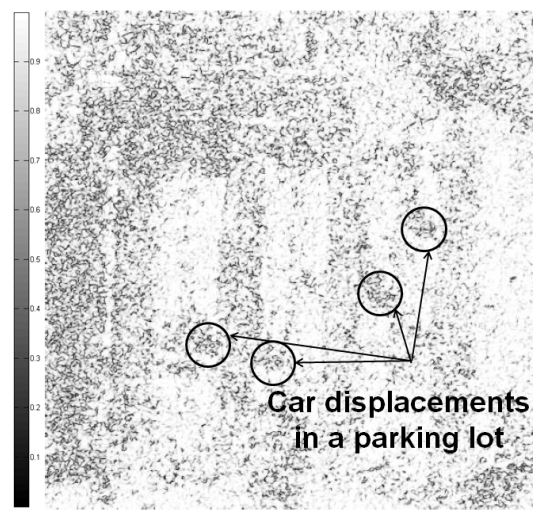

(a) $\left|\hat{\rho}_{c}\right|$ (state of the art)

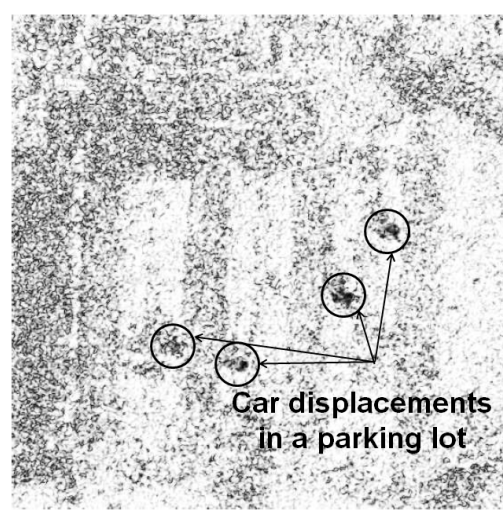

(b) $\left|\hat{\rho}_{a}\right|$

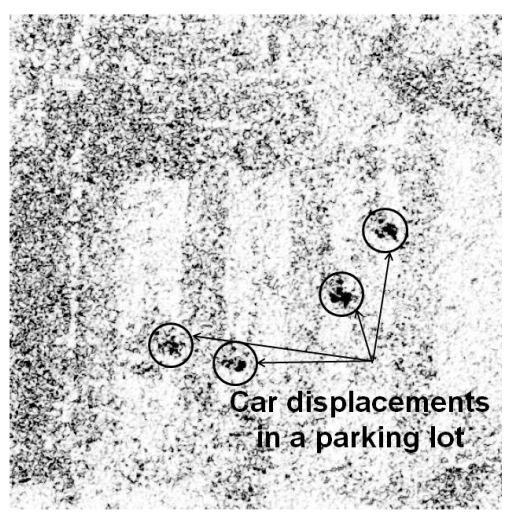

(c) Two-stage

Fig. 16: Change detection images formed using AFRL SAR data with $N=5$

several footprints that originate on a road. As in the previous example, there are false alarms with considerable variation. The faint tracks that are partially disconnected in this kind of environment make the problem of track detection especially difficult. The two-stage result provides the highest contrast with sharpest foot tracks in Fig. 15.

Fig. 16 shows the results of the three change detection methods on a SAR pair of a parking lot scene, where detecting object displacements amid numerous false alarms is important. As uninteresting changes due to the environment are technically a type of change, the coherence of those pixels are decreased along with the pixels for the changes of interest. However, the increase in probability of detection caused by underestimation of $\left|\hat{\rho}_{a}\right|$ when $\sigma_{f} \neq \sigma_{g}$ overweighs the slight increase in false alarms due to lower coherence estimates of uninteresting change and noise. Again, the contrast is emphasized in the two-stage method, making the change even sharper.

\section{CONCLUSiOnS AND Future Work}

In this work, we have derived a two-stage test statistic for SAR change detection. This method is based on the use of the intensity change detector $\hat{R}$ using a sample variance ratio followed by the coherent change detector $\left|\hat{\rho}_{a}\right|$ that assumes equal population variances. The proposed method not only has been shown to have a better change detection performance over the current stateof-the-art change detector $\left|\hat{\rho}_{c}\right|$, but also over either $\hat{R}$ or $\left|\hat{\rho}_{a}\right|$, alone. The significant improvement in change detection performance was achieved due to the following reasons. 
1) When $R=1$ and $|\rho|$ is low, $\left|\hat{\rho}_{a}\right|$ yields significantly less mean squared error than $\left|\hat{\rho}_{c}\right|$, and when $R=1$ and $|\rho|$ is high, $\left|\hat{\rho}_{a}\right|$ yields slightly more error than $\left|\hat{\rho}_{c}\right|$, but the magnitude of the difference in estimation error is negligible, and

2) When $R \neq 1$, the direct distributional properties assign lower estimated coherence to $\left|\hat{\rho}_{a}\right|$ than $\left|\hat{\rho}_{c}\right|$, therefore if $|\rho|$ is low, true change is more likely to be detected, but if $|\rho|$ is high, more false alarms are likely to be detected as change. However, the constraint of $p\left(\left|\hat{\rho}_{a}\right|, \hat{R}\right)$ shows that such events are unlikely with low $\hat{R}$.

The improved performance of the two-stage detector compared to other detectors is investigated in terms of ROC curves and visual inspection of the resulting change detection images.

We have derived the theoretical probability density function of the two-stage change detector. In our initial study, we focused on the development of a heuristic approach to choosing thresholds and use of empirical analysis to demonstrate potential due to the integral of the joint probability density function being non-trivial to be solved analytically. A detailed study using the probability density function to explore the inherent behavior of the detector will be included in the future work.

\section{APPENDIX}

\section{DERIVATION OF $p\left(\left|\hat{\rho}_{a}\right|, \hat{R}\right)$}

The joint density of the magnitude of the estimator $\left|\hat{\rho}_{a}\right|$ and the sample variance ratio $\hat{R}$ can be computed by extending the method of [7] as follows. First, two auxiliary variables $v=A_{11}+A_{22}$ and $w=A_{22}$ are introduced. Note that $A_{11}=v-w, A_{22}=w, \operatorname{Re}\left(A_{12}\right)=$ $\operatorname{Re}\left(\hat{\rho}_{a}\right) v / 2, \operatorname{Im}\left(A_{12}\right)=\operatorname{Im}\left(\hat{\rho}_{a}\right) v / 2$, and the determinant of the Jacobian of the transformation $\left(A_{11}, A_{22}, \operatorname{Re}\left(A_{12}\right), \operatorname{Im}\left(A_{12}\right)\right) \rightarrow\left(\operatorname{Re}\left(\hat{\rho}_{a}\right), \operatorname{Im}\left(\hat{\rho}_{a}\right), v, w\right)$ is $\left|J_{1}\right| \equiv\left|\frac{\partial\left(A_{11}, A_{22}, \operatorname{Re}\left(A_{12}\right), \operatorname{Im}\left(A_{12}\right)\right.}{\partial\left(\operatorname{Re}\left(\hat{\rho}_{a}\right), \operatorname{Im}\left(\hat{\rho}_{a}\right), v, w\right)}\right|=$ $(v / 2)^{2}$. It follows from (4) that

$$
\begin{aligned}
p\left(\operatorname{Re}\left(\hat{\rho}_{a}\right), \operatorname{Im}\left(\hat{\rho}_{a}\right), v, w\right)= & \left|J_{1}\right| p\left(A_{11}, A_{22}, \operatorname{Re}\left(A_{12}\right), \operatorname{Im}\left(A_{12}\right)\right) \\
= & \left|J_{1}\right| p\left(v-w, w, \operatorname{Re}\left(\hat{\rho}_{a}\right) v / 2, \operatorname{Im}\left(\hat{\rho}_{a}\right) v / 2\right) \\
= & (v / 2)^{2} K_{1}\left[(v-w) w-\left(\left|\hat{\rho}_{a}\right| v / 2\right)^{2}\right]^{N-2} \\
& \cdot \exp \left[-v \gamma^{11}+w\left(\gamma^{11}-\gamma^{22}\right)-v \operatorname{Re}\left(\gamma^{12} \overline{\hat{\rho}}_{a}\right)\right]
\end{aligned}
$$

where

$$
K_{1}=\left[\pi \Gamma(N) \Gamma(N-1) \sigma_{f}^{2 N} \sigma_{g}^{2 N}\left(1-|\rho|^{2}\right)^{N}\right]^{-1}
$$


$\gamma^{j k}$ is the $(j, k)$ th element of $\Sigma^{-1}$, and the density vanishes outside the region $0 \leq w \leq v<\infty$, $\left|\hat{\rho}_{a}\right| \leq 2\left[(w / v)-(w / v)^{2}\right]^{\frac{1}{2}}$.

Next, consider the second change of variables $\left(\operatorname{Re}\left(\hat{\rho}_{a}\right), \operatorname{Im}\left(\hat{\rho}_{a}\right), v, w\right) \rightarrow\left(\operatorname{Re}\left(\hat{\rho}_{a}\right), \operatorname{Im}\left(\hat{\rho}_{a}\right), \hat{R}, w\right)$ where $\hat{R}=v / w-1$. The Jacobian of this transformation is $\left|J_{2}\right| \equiv\left|\frac{\partial v}{\partial \hat{R}}\right|=w$, and the density expressed in the new variables is

$$
\begin{aligned}
p\left(\operatorname{Re}\left(\hat{\rho}_{a}\right), \operatorname{Im}\left(\hat{\rho}_{a}\right), \hat{R}, w\right)= & \left|J_{2}\right| p\left(\operatorname{Re}\left(\hat{\rho}_{a}\right), \operatorname{Im}\left(\hat{\rho}_{a}\right), v, w\right) \\
= & \left|J_{2}\right| p\left(\operatorname{Re}\left(\hat{\rho}_{a}\right), \operatorname{Im}\left(\hat{\rho}_{a}\right), w(\hat{R}+1), w\right) \\
= & K_{1} w^{2 N-1}\left(\frac{\hat{R}+1}{2}\right)^{2}\left[\hat{R}-\left|\hat{\rho}_{a}\right|^{2} \frac{(\hat{R}+1)^{2}}{4}\right]^{N-2} \exp (-w \phi) \\
& \left(\left|\hat{\rho}_{a}\right| \leq\left[4 \hat{R} /(\hat{R}+1)^{2}\right]^{\frac{1}{2}}, 0 \leq \hat{R}<\infty, 0 \leq w<\infty\right)
\end{aligned}
$$

where

$$
\phi=\left[(\hat{R}+1) \gamma^{11}+\left(\gamma^{22}-\gamma^{11}\right)+(\hat{R}+1) \operatorname{Re}\left(\gamma^{12} \overline{\hat{\rho}}_{a}\right)\right] .
$$

Using the integral solved in [2],

$$
\int_{0}^{\infty} \omega^{n-1} \exp [-\omega \mu] d \omega=\frac{1}{\mu^{n}} \Gamma(n),
$$

we next obtain $p\left(\operatorname{Re}\left(\hat{\rho}_{a}\right), \operatorname{Im}\left(\hat{\rho}_{a}\right), \hat{R}\right)$ by integrating $p\left(\operatorname{Re}\left(\hat{\rho}_{a}\right), \operatorname{Im}\left(\hat{\rho}_{a}\right), \hat{R}, w\right)$ over $w$,

$$
\begin{aligned}
p\left(\operatorname{Re}\left(\hat{\rho}_{a}\right), \operatorname{Im}\left(\hat{\rho}_{a}\right), \hat{R}\right)= & \int_{0}^{\infty} p\left(\operatorname{Re}\left(\hat{\rho}_{a}\right), \operatorname{Im}\left(\hat{\rho}_{a}\right), \hat{R}, w\right) d w \\
= & K_{1}\left(\frac{\hat{R}+1}{2}\right)^{2}\left[\hat{R}-\left|\hat{\rho}_{a}\right|^{2} \frac{(\hat{R}+1)^{2}}{4}\right]^{N-2} \phi^{-2 N} \Gamma(2 N) \\
= & K_{1} \frac{1}{4(\hat{R}+1)^{2}}\left[\frac{1}{\hat{R}+1}\left(1-\frac{1}{\hat{R}+1}\right)-\frac{\left|\hat{\rho}_{a}\right|^{2}}{4}\right]^{N-2} \\
& \cdot\left[\gamma^{11}+\frac{\gamma^{22}-\gamma^{11}}{\hat{R}+1}+\operatorname{Re}\left(\gamma^{12} \overline{\hat{\rho}}_{a}\right)\right]^{-2 N} \Gamma(2 N) \\
& \left(\left|\hat{\rho}_{a}\right| \leq\left[4 \hat{R} /(\hat{R}+1)^{2}\right]^{\frac{1}{2}}, 0 \leq \hat{R}<\infty\right)
\end{aligned}
$$

The third change of variables $\left(\operatorname{Re}\left(\hat{\rho}_{a}\right), \operatorname{Im}\left(\hat{\rho}_{a}\right), \hat{R}\right) \rightarrow\left(\left|\hat{\rho}_{a}\right|, \theta_{\hat{\rho}_{a}}, \hat{R}\right)$ facilitates transformation of (25) to polar coordinates. Here, $\operatorname{Re}\left(\hat{\rho}_{a}\right)=\left|\hat{\rho}_{a}\right| \cos \left(\theta_{\hat{\rho}_{a}}\right), \operatorname{Im}\left(\hat{\rho}_{a}\right)=\left|\hat{\rho}_{a}\right| \sin \left(\theta_{\hat{\rho}_{a}}\right)$, and the determinant of the Jacobian matrix is $\left|J_{3}\right| \equiv\left|\frac{\partial\left(\operatorname{Re}\left(\hat{\rho}_{a}\right), \operatorname{Im}\left(\hat{\rho}_{a}\right)\right)}{\partial\left(\left|\hat{\rho}_{a}\right|, \theta_{\hat{\rho}_{a}}\right)}\right|=\left|\hat{\rho}_{a}\right|$. Using the explicit expressions 
for $\gamma^{11}, \gamma^{22}$, and $\gamma^{12}$, the density in the new coordinates can be represented as

$$
\begin{aligned}
p\left(\left|\hat{\rho}_{a}\right|, \theta_{\hat{\rho}_{a}}, \hat{R}\right)= & \left|J_{3}\right| p\left(\operatorname{Re}\left(\hat{\rho}_{a}\right), \operatorname{Im}\left(\hat{\rho}_{a}\right), \hat{R}\right) \\
= & \left|J_{3}\right| p\left(\left|\hat{\rho}_{a}\right| \cos \left(\theta_{\hat{\rho}_{a}}\right),\left|\hat{\rho}_{a}\right| \sin \left(\theta_{\hat{\rho}_{a}}\right), \hat{R}\right) \\
= & \frac{\left(1-|\rho|^{2}\right)^{N} \Gamma(2 N)}{\pi \Gamma(N) \Gamma(N-1)} \frac{\left|\hat{\rho}_{a}\right|}{4(\hat{R}+1)^{2}}\left[\frac{1}{\hat{R}+1}\left(1-\frac{1}{\hat{R}+1}\right)-\frac{\left|\hat{\rho}_{a}\right|^{2}}{4}\right]^{N-2} \\
& \cdot\left[\frac{\sigma_{g}}{\sigma_{f}}+\frac{1}{(\hat{R}+1)} \frac{\sigma_{f}^{2}-\sigma_{g}^{2}}{\sigma_{f} \sigma_{g}}-\left|\hat{\rho}_{a}\right||\rho| \cos \left(\theta_{\hat{\rho}_{a}}-\theta_{\rho}\right)\right]^{-2 N} \\
& \left(\left|\hat{\rho}_{a}\right| \leq\left[4 \hat{R} /(\hat{R}+1)^{2}\right]^{\frac{1}{2}}, 0 \leq \hat{R}<\infty,-\pi \leq \theta_{\hat{\rho}_{a}} \leq \pi\right) .
\end{aligned}
$$

As the final step, we integrate $p\left(\left|\hat{\rho}_{a}\right|, \theta_{\hat{\rho}_{a}}, \hat{R}\right)$ with respect to $\theta_{\hat{\rho}_{a}}$, following the analytical expression,

$$
\int_{-\pi}^{\pi}[p+q \cos (x)]^{-2 n} d x=2 \pi(p-q)^{-2 n} F_{1}\left(\frac{1}{2}, 2 n, 1, \frac{2 q}{q-p}\right) \quad(p>q) .
$$

In our case,

$$
\begin{aligned}
p & =\frac{\sigma_{g}}{\sigma_{f}}+\frac{1}{(\hat{R}+1)} \frac{\sigma_{f}^{2}-\sigma_{g}^{2}}{\sigma_{f} \sigma_{g}}=\frac{\sigma_{g}^{2} \hat{R}+\sigma_{f}^{2}}{(\hat{R}+1) \sigma_{f} \sigma_{g}} \\
q & =-\left|\hat{\rho}_{a}\right||\rho|,
\end{aligned}
$$

therefore, $p>q$ holds. This leads to the joint density function of $\left|\hat{\rho}_{a}\right|$ and $\hat{R}$,

$$
\begin{aligned}
p\left(\left|\hat{\rho}_{a}\right|, \hat{R}\right)= & \frac{\left(1-|\rho|^{2}\right)^{N} \Gamma(2 N)}{\Gamma(N) \Gamma(N-1)} \frac{\left|\hat{\rho}_{a}\right|}{2(\hat{R}+1)^{2}}\left[\frac{1}{\hat{R}+1}\left(1-\frac{1}{\hat{R}+1}\right)-\frac{\left|\hat{\rho}_{a}\right|^{2}}{4}\right]^{N-2} \\
& \cdot\left[\left|\hat{\rho}_{a}\right||\rho|+\frac{\sigma_{g}^{2} \hat{R}+\sigma_{f}^{2}}{(\hat{R}+1) \sigma_{f} \sigma_{g}}\right]^{-2 N} F_{1}\left(\frac{1}{2}, 2 N, 1, \frac{2\left|\hat{\rho}_{a}\right||\rho|}{\left|\hat{\rho}_{a}\right||\rho|+\frac{\sigma_{g}^{2} \hat{R}+\sigma_{f}^{2}}{(\hat{R}+1) \sigma_{f} \sigma_{g}}}\right)
\end{aligned}
$$

subject to

$$
\left|\hat{\rho}_{a}\right| \leq\left[4 \hat{R} /(\hat{R}+1)^{2}\right]^{\frac{1}{2}}, 0 \leq \hat{R}<\infty
$$

\section{ACKNOWLEDGMENT}

We would like to thank Michael Hurley for his careful review of this journal and many constructive comments, and Stephen Taylor for his thoughtful comments and analysis. We also thank AFRL/RYA for providing a set of X-band SAR imagery. 


\section{REFERENCES}

[1] D. Massonnet and K.L. Feigl, "Radar interferometry and its application to changes in the earth's surface," Reviews of Geophysics, 1998.

[2] D.G. Corr and A. Rodrigues, "Coherent change detection of vehicle movements," IEEE International Geosci. Remote Sensing, pp. 2451-2453, 1998.

[3] M.L. Williams and M. Preiss, "Physics-based predictions for coherent change detection using x-band synthetic aperture radar," Eurasip Journal of Applied Signal Processing, vol. 2005, no. 20, pp. 3243-3258, 2005.

[4] A. Marino, S.R. Cloude, and J.M. Lopez-Sanchez, “A new polarimetric change detector in radar imagery,” IEEE Trans. Geosci. Remote Sensing, vol. 51, pp. 2986-3000, 2013.

[5] M. Liu, H. Zhang, C. Wang, and F. Wu, "Change detection of multilook polarimetric sar images using heterogeneous clutter models," IEEE Trans. Geosci. Remote Sensing, vol. 52, pp. 7483-7494, 2014.

[6] M. Preiss, D.A. Gray, and N.J.S. Stacy, "Detecting scene changes using synthetic aperture radar interferometry," IEEE Trans. Geosci. Remote Sensing, vol. 44, pp. 2041-2054, 2006.

[7] T. Berger, "On the correlation coefficient of a bivariate, equal variance, complex Gaussian sample," Ann. Math. Stat., vol. 43, pp. 2000-2003, 1972.

[8] R. Touzi and A. Lopes, "Statistics of the stokes parameters and of the complex coherence parameters in one-look and multilook speckle fields," IEEE Trans. Geosci. Remote Sensing, vol. 34, pp. 764-773, 1996.

[9] M. Liao, L. Jiang, H. Lin, B. Huang, and J. Gong, "Urban change detection based on coherence and intensity characteristics of sar imagery," Photogrammetric Engineering and Remote Sensing, 2008.

[10] M. Cha, R.D. Phillips, and P.J. Wolfe, "Test statistics for synthetic aperture radar coherent change detection," in IEEE SSP Workshop, 2012.

[11] N. R. Goodman, "Statistical analysis based on a certain multivariate complex gaussian distribution," in "Ann. Math. Stat., 1963, vol. 34, pp. 152-177.

[12] M. Preiss and N.J.S. Stacy, "Coherent change detection: Theoretical description and experimental results," Defence Science and Technology Organisation, Australia, 2006.

[13] Z. Wei, G. Jian, and W. Jie, "Change detection of concealed targets using repeat-pass sar images," in IEEE APSAR, 2007.

[14] R.K. Andrew and R.L Kirlin, "An equality test for variances of two complex correlated gaussian processes," IEEE Trans. Signal Process., vol. 48, pp. 2452-2454, 2000.

[15] T. Medkour and A.T. Walden, "Attenuation estimation from correlated sequences," IEEE Trans. Signal Process., vol. 50, pp. 378-383, 2007.

[16] T. Medkour and A.T. Walden, "A variance equality test for two correlated complex gaussian variables with application to spectral power comparison,” IEEE Trans. Signal Process., vol. 55, pp. 881-888, 2007.

[17] I.S. Gradshteyn and I.M. Ryzhik, Table of Integrals, Series and Products, New York: Academic, 1980.

[18] S.M. Scarborough, L. Gorham, M.J. Minardi, U.K. Majumder, M.G. Judge, L. Moore, L. Novak, S. Jaroszewksi, L. Spoldi, and A. Pieramico, "A challenge problem for SAR change detection and data compression," Proc. SPIE, vol. 7699, pp. 76990 U, 2010. 